\title{
SCATTERING MATRICES AND SCATTERING GEODESICS OF LOCALLY SYMMETRIC SPACES
}

\author{
BY LIZHEN JI AND MACIEJ ZWORSKI
}

\begin{abstract}
Let $\Gamma / X$ be a $\mathbb{Q}$-rank one locally symmetric space. We describe the frequencies of oscillation of scattering matrices on $\Gamma / X$ in the energy variable in terms of sojourn times of scattering geodesics. Scattering geodesics are the geodesics which move to infinity in both directions and are distance minimizing near both infinities. The sojourn time of a scattering geodesic is the time it spends in a fixed compact region. The frequencies of oscillation come from the singularities of the Fourier transforms of scattering matrices and we show that these occur at sojourn times of scattering geodesics on the locally symmetric space. This generalizes a result of Guillemin obtained in the case of finite volume noncompact Riemann surfaces. ๑ 2001 Éditions scientifiques et médicales Elsevier SAS
\end{abstract}

RÉSUMÉ. - Soit $\Gamma / X$ un espace localement symétrique de rang 1 sur $\mathbb{Q}$. Nous décrivons les fréquences d'oscillations de la matrice de scattering de $\Gamma / X$ dans les variables d'énergie. Définissons pour cela les géodésiques de scattering de l'espace localement symétrique comme les géodésiques atteignant l'infini à leurs deux extrémités, et qui sont minimisantes au voisinage de l'infini. Appelons temps de séjour d'une telle géodésique le temps qu'elle passe dans un domaine compact fixé. Nous prouvons que les singularités de la transformée de Fourier (relativement au paramètre d'énergie) de la matrice de scattering sont contenues dans l'ensemble des temps de séjour, et décrivons la nature de ces singularités. Cela généralise un résultat de Guillemin obtenu dans le cas des surfaces de Riemann non compactes de volume fini. @ 2001 Éditions scientifiques et médicales Elsevier SAS

\section{Introduction}

The purpose of this paper is to describe the frequencies of oscillation (in the energy variable) of scattering matrices on $\mathbb{Q}$-rank one locally symmetric spaces $\Gamma \backslash X$. In other words, we describe the singularities of the Fourier transforms (with respect to the energy parameter) of scattering matrices and show that they occur at sojourn times of scattering geodesics on the locally symmetric space. This generalizes a result of Guillemin [9] obtained in the case of finite volume noncompact Riemann surfaces. In the case when $\mathbb{Q}$-rank is greater than one, the method of this paper would also give some results (see Remarks at the end of Section 3) but we restrict ourselves to a simpler case at this early stage.

Scattering geodesics are the geodesics which move to infinity in both directions and are distance minimizing near both infinities - see Proposition 2.5 and Definition 2.4 for a more direct geometric description. The sojourn time of a scattering geodesic is, roughly speaking, the time it spends in a fixed compact region.

Let $X=G / K$ be a symmetric space of noncompact type, and $\Gamma \subset G$ an arithmetic discrete subgroup of finite covolume. Then $\Gamma \backslash X$ is a locally symmetric space of finite volume. When $\Gamma \backslash X$ is noncompact, it has both discrete and continuous spectra. The continuous spectrum is 
given by the Eisenstein series, and the constant terms of the Eisenstein series along rational parabolic subgroups are described by the scattering matrices. For general $\Gamma \backslash X$, the Eisenstein series and scattering matrices were studied by Langlands in [18] (see Lemma 3.5 and (3.3) below). Roughly speaking, the scattering matrices measure the density of the continuous spectrum, and their analytic properties and functional equations play an important role in the Langlands' program (see [19]).

The scattering matrices in the $\mathbb{Q}$-rank one case take a particularly simple form. Specifically, in the $\mathbb{Q}$-rank case, there is a one-to-one correspondence between the set of ends of $\Gamma \backslash X$ and the set of $\Gamma$-conjugacy classes of rational parabolic subgroups. Topologically, each end is a cylinder. For each end of the space, let us denote the associated parabolic subgroup by $P$. We associate to it a compact locally symmetric space, $\Gamma_{X_{P}} \backslash X_{P}$, of smaller dimension (see Proposition 2.3). Then, a section of the topological end associated with $P$ is a fiber bundle over $\Gamma_{X_{P}} \backslash X_{P}$, with fiber equal to $\Gamma_{N_{P}} \backslash N_{P}$, a compact quotient of a unipotent group $N_{P}$. Let $P_{1}, \ldots, P_{n}$ be a set of representatives of $\Gamma$-conjugacy classes of rational parabolic subgroups. For each $\mu \in \mathbb{R}$ we then consider the direct sum of the $\mu$-eigenspaces of the Laplacians on $\Gamma_{X_{P_{i}}} \backslash X_{P_{i}}, i=1, \ldots, n$. The scattering matrix $S^{\mu}$ (also denoted by $c_{\mu}$ below) is a unitary operator on that space. For $\mu=0$, the dimension of the space is equal to the number of the ends of $\Gamma \backslash X$, and it is non-zero only for a discrete set of $\mu$ 's in $\bigcup_{i=1}^{n} \operatorname{Spec}\left(\Gamma_{P_{i}} \backslash X_{P_{i}}\right)$. For a particular orthonormal basis of eigenvectors corresponding to $\mu$,

$$
\phi_{1}^{\mu}, \ldots, \phi_{K(\mu)}^{\mu}, \quad \phi_{j}^{\mu} \in L^{2}\left(\Gamma_{X_{P_{k(j)}}} \backslash X_{P_{k(j)}}\right),
$$

where $K(\mu)$ is the dimension of the direct sum of the eigenspaces, and $k(j) \in\{1, \ldots, n\}$ is the unique index such that $\phi_{j}^{\mu}$ is an eigenfunction on $\Gamma_{X_{P_{k(j)}}} \backslash X_{P_{k(j)}}$, we have the following expression for the scattering matrix $S^{\mu}(\lambda)$ :

$$
S^{\mu}(\lambda)=\left\{s_{i j}^{\mu}(-\mathrm{i} \lambda)\right\}_{1 \leqslant i, j \leqslant K(\mu)} .
$$

The operator $S^{\mu}(\lambda)$ is meromorphic in $\lambda \in \mathbb{C}$ (we are in the $\mathbb{Q}$-rank one case) and we are interested in values of $\lambda \in \mathbb{R}$, corresponding to the continuous spectrum.

Using this notation we can now state our results. The detailed definitions of all other objects appearing in the statement will be given in Section 2.

THEOREM 1. - Let $\Gamma \backslash X$ be a $\mathbb{Q}$-rank one locally symmetric space. For $l, m \in\{1, \ldots, n\}$, let $\mathcal{T}_{\text {lm }} \subset \mathbb{R}$ be the set of sojourn times of scattering geodesics between the ends corresponding to parabolic subgroups $P_{l}$ and $P_{m}$. Then $\mathcal{T}_{l m}$ is discrete and for each $T \in \mathcal{T}_{l m}$ the corresponding scattering geodesics form a smooth family parametrized by a common finite cover $X_{T, m l}$ of $\Gamma_{X_{P_{l}}} \backslash X_{P_{l}}$ and $\Gamma_{X_{P_{m}}} \backslash X_{P_{m}}$ with projections

$$
\begin{gathered}
X_{T, m l} \\
\Gamma_{X_{P_{l}} \backslash X_{P_{l}}} \stackrel{\pi_{m, T}}{\Gamma_{X_{P_{m}}} \backslash X_{P_{m}}}
\end{gathered}
$$

defined in (2.4).

If we think of the geometric Theorem 1 as a classical statement, the next theorem provides the corresponding quantum property of locally symmetric spaces. It partially answers [16, Question 13.17.2].

To formulate it, we need to discuss the structure of $\Gamma \backslash X$ more - it will be carefully reviewed in Section 2. As recalled above, to each infinite end we associate a parabolic subgroup $P$, which 
admits the Langlands decomposition $N_{P} M_{P} A_{P}$. Let $\Phi^{+}\left(P, A_{P}\right)$ be the set of the roots in $\mathfrak{a}_{P}^{*}$ of the adjoint action of $A_{P}$ on $N_{P}$. We then define

$$
2 \rho=2 \rho_{P}=\text { sum of the roots in } \Phi^{+}\left(P, A_{P}\right),
$$

where we add with multiplicities equal to the dimensions of the root spaces. In the $\mathbb{Q}$-rank one case, $\operatorname{dim} A_{P}=1$, and $\mathfrak{a}_{P}^{*}$ can be identified with $\mathbb{R}$ such that the norm on $\mathfrak{a}_{P}^{*}$ defined by the Killing form is equal to the standard Euclidean norm of $\mathbb{R}$ and the roots in $\Phi^{+}\left(P, A_{P}\right)$ and hence $\rho_{P}$ are identified with positive numbers. Then $\rho$ is the same positive number for all rational parabolic subgroups $P$. The group $N_{P}$ can be identified with the Riemannian submanifold $N_{P} x_{0}$ in $X$, where $x_{0}=K \in X=G / K$. We then have a natural quotient $\Gamma_{N_{P}} \backslash N_{P}, \Gamma_{N_{P}}=\Gamma \cap N_{P}$, also equipped with a Riemannian density.

THEOREM 2.- Let $\Gamma \backslash X$ be a $\mathbb{Q}$-rank 1 locally symmetric space, and $s_{i j}^{\mu}(\lambda)$ be the components of the scattering matrix for the eigenvalue $\mu$ with respect to the orthonormal basis $\phi_{1}^{\mu}, \ldots, \phi_{K(\mu)}^{\mu}$ as above. Let $l=k(i), m=k(j) \in\{1, \ldots, n\}$ be defined as in 1.1 , and $\mathcal{T}_{l m}$ the set of sojourn times between the ends of $P_{l}$ and $P_{m}$ as in Theorem 1. Then

$$
\operatorname{sing} \operatorname{supp} \hat{s}_{i j}^{\mu} \subset \mathcal{T}_{l m},
$$

and, more precisely, $\hat{s}_{i j}^{\mu}(t)$ is equal to

$$
\sum_{T \in \mathcal{T}_{l m}} \mathrm{e}^{-\rho T} \frac{(2 \pi)^{-e_{T}^{m l} / 2+1}}{v_{m} v_{l}}\left(\int_{X_{T, m l}} \pi_{l, T}^{*} \phi_{i} \pi_{m, T}^{*} \phi_{j}\right) \sum_{ \pm}(t-T \pm \mathrm{i} 0)^{-1 / 2-e_{T}^{m l} / 2}\left(1+g_{T}^{m l}(t)\right),
$$

where $g_{T}^{m l}$ are smooth in a neighbourhood of $T, g_{T}^{m l}(T)=0$,

$$
e_{T}^{m l}=\operatorname{dim} X_{T, m l},
$$

$v_{p}$ is the volume of $\Gamma_{N_{P_{p}}} \backslash N_{P_{p}}$ with respect to the induced Riemannian metric, $p=l, m, \rho$ is given by (1.3), and the integration over $X_{T, m l}$ is with respect to the Riemannian density.

The statement of the theorem can be transformed into a statement about the frequencies of oscillations of the scattering matrix. Suppose that $\phi \in \mathcal{C}_{\mathrm{c}}^{\infty}(\mathbb{R})$. Then

$$
\begin{gathered}
s_{i j}^{\mu} * \hat{\phi}(\lambda)=\sum_{T \in \operatorname{supp} \phi \cap \mathcal{T}_{l m}} a_{T, \phi}^{m l}(\lambda) \mathrm{e}^{-\mathrm{i} T \lambda}+\mathcal{O}_{\phi}\left(\lambda^{-\infty}\right), \\
a_{T, \phi}^{m l}(\lambda)=\sum_{k \geqslant 0} a_{T, \phi, k}^{m l} \lambda^{e_{T}^{m l} / 2-1 / 2-k},
\end{gathered}
$$

with coefficients easily determined from the leading coefficient in Theorem 2 and the Taylor expansion of $g_{T}^{m l}$ at $T$. It is possible (see (1.5) below) that a finer analysis based on less general methods will give an expansion without the need for a cut-off $\phi$.

As mentioned above, when $X=S L(2, \mathbb{R}) / S O(2)$ and $\Gamma$ is a co-finite discrete subgroup of $S L(2, \mathbb{R})$, the result is due to Guillemin [9], [10]. In that case the only contribution comes from $\mu=0$, the spaces $X_{T, m l}$ reduce to points and $m, l$ range from one to the number of cusp ends. The argument of [9] was a direct computation, and in a more general case of a non-constant curvature, an application of the method coming from Euclidean scattering. It was based on the 
construction of wave operators using the theory of Fourier Integral Operators. A nice feature of the Riemann surface case is that the same theorem holds if a compactly supported metric perturbation is introduced. In Theorem 2 that is not the case. The spectral decomposition changes dramatically when the structure of a locally symmetric space is perturbed and we do not know a proper analogue of the quantum statement. Similarly, the structure of scattering geodesics will also change dramatically under perturbations: for example, the set of sojourn times may not be discrete anymore.

To compare with (1.4), we recall Guillemin's explicit expansion in the case of $\Gamma \backslash \mathbb{H}^{2}$ :

$$
s_{i j}(\lambda)=c(\lambda) \sum_{T \in \mathcal{T}_{i j}} \mathrm{e}^{-T / 2} \mathrm{e}^{-\mathrm{i} \lambda T}, \quad c(\lambda)=\int_{-\infty}^{\infty} \frac{\mathrm{d} q}{\left(1+q^{2}\right)^{1 / 2+\mathrm{i} \lambda}},
$$

where now we only have $\mu=0$ and $k(i)=i, k(j)=j$, index the cusps.

In this paper, rather than to follow [9] and construct the wave operators or to study Eisenstein series explicitly, we adapt an observation of Zelditch [25]. It was in turn inspired by Kuznecov sum formula: the real part of the scattering matrix can be given as an integral of the wave group over horocycles (see Proposition 4.1). That gives an easy way for understanding the singularities of the Fourier transform of the real part of the scattering matrix [25, (2.10),(2.12)]. We observe (Lemma 5.1) that we can also obtain singularities of the Fourier transform of the scattering matrix itself that way. Once the geometry is understood (Theorem 1 and Section 2), the same method applies in the case studied here.

We also point out that in the Euclidean setting, sojourn times are an old object of classical (and semi-classical) scattering. For that, and for pointers to recent work, we refer to [22].

\section{Geometry of locally symmetric spaces}

In this section, we define arithmetic subgroups, locally symmetric spaces and their geometry at infinity. The geometry at infinity is needed to understand scattering geodesics and hence their sojourn time, and is also crucial to understanding the continuous spectrum and the spectral decomposition. Though we will mainly study locally symmetric spaces of $\mathbb{Q}$-rank 1 in $\S 4$ and $\S 5$, we formulate some results in $\S 2$ and $\S 3$ without this restriction. An important class of $\mathbb{Q}$-rank 1 locally symmetric spaces consists of Hilbert modular varieties (see [7]).

We begin by recalling some notions from algebraic group theory. The references are [1-4]. Let $\mathbf{G}=\mathbf{G}(\mathbb{C})$ be a connected semisimple linear algebraic group defined over $\mathbb{Q}, G=\mathbf{G}(\mathbb{R})$ the real locus of $\mathbf{G}$, which is a semisimple Lie group with finitely many connected components. Let $K \subset G$ be a maximal compact subgroup. The Killing form of the Lie algebra of $G$ defines a $G$-invariant Riemannian metric on $X=G / K$, and $X$ is a Riemannian symmetric space of noncompact type.

Definition 2.1. - A subgroup $\Gamma$ of the rational locus $\mathbf{G}(\mathbb{Q})$ of $\mathbf{G}$ is called an arithmetic subgroup if under an embedding $\mathbf{G} \rightarrow G L(n, \mathbb{C})$ which is defined over $\mathbb{Q}, \Gamma$ is commensurable to $G \cap G L(n, \mathbb{Z})$.

The embedding $\mathbf{G} \rightarrow G L(n, \mathbb{C})$ exists because $\mathbf{G}$ is by definition a linear algebraic group defined over $\mathbb{Q}$. The class of arithmetic subgroups is well-defined and does not depend on the embedding $\mathbf{G} \rightarrow G L(n, \mathbb{C})$. Furthermore, a result of Selberg [24, Lemma 8] says that any arithmetic subgroup has a subgroup of finite index which is torsion free, and which is also clearly arithmetic. 
Any torsion free arithmetic subgroup $\Gamma$ acts properly discontinuously and fixed point freely on $X$, and the quotient $\Gamma \backslash X$ is a locally symmetric space of finite volume. In the following, we assume that $\Gamma$ is torsion free, and $\Gamma \backslash X$ is noncompact . A good example to keep in mind while reading this paper is $\Gamma=\Gamma_{n}=\{g \in S L(n, \mathbb{Z}) \mid g \equiv I d \bmod n\}$, and $X$ is the space of positive definite unimodular matrices.

The first natural question is to understand the geometry of $\Gamma \backslash X$ near infinity, and hence to see how geodesics and points of $\Gamma \backslash X$ go to infinity. For this purpose, we need to recall the reduction theory, in particular, rational parabolic subgroups and their Langlands decomposition.

A closed subgroup $\mathbf{P}$ of $\mathbf{G}$ is called a parabolic subgroup if $\mathbf{P}$ contains a maximal connected solvable subgroup, i.e., a Borel subgroup, of $\mathbf{G}$. This condition is equivalent to the condition that the quotient $\mathbf{P} \backslash \mathbf{G}$ is compact (or a projective variety, to be precise). If $\mathbf{P}$ is defined over $\mathbb{Q}, \mathbf{P}$ is called a rational parabolic subgroup.

For any rational parabolic subgroup $\mathbf{P}$, let $\mathbf{N}_{P}$ be the greatest unipotent normal subgroup of $\mathbf{P}$, which is called the unipotent radical of $\mathbf{P}$ and is defined over $\mathbb{Q}$. When $\mathbf{G}=S L(n)$, the subgroup of upper triangular matrices is a rational parabolic subgroup $\mathbf{P}$, and $\mathbf{N}_{P}$ is the subgroup of upper triangular matrices with 1 on the diagonal, i.e., unipotent upper triangular matrices. The quotient $\mathbf{N}_{P} \backslash \mathbf{P}$ is called the Levi quotient of $\mathbf{P}$ and is a reductive algebraic group defined over $\mathbb{Q}$, denoted by $\mathbf{L}_{P}$.

To decompose $\mathbf{P}$, we need to lift $\mathbf{L}_{P}$ to a subgroup of $\mathbf{P}$ (see $[4, \S 1]$ ). Let $x_{0}=K \in X=G / K$ be a fixed basepoint. Then there is a unique lift $i_{x_{0}}: \mathbf{L}_{P} \rightarrow \mathbf{P}$ such that $i_{x_{0}}\left(\mathbf{L}_{P}\right)$ is a closed subgroup of $\mathbf{G}$ stable under the Cartan involution $\theta$ associated with $K$. In the following, $i_{x_{0}}$ is also denoted by $i_{0}$ for simplicity.

Let $N_{P}=\mathbf{N}_{P}(\mathbb{R}), P=\mathbf{P}(\mathbb{R}), L=\mathbf{L}(\mathbb{R})$. Let $\mathbf{S}_{P}$ be the maximal torus in $\mathbf{L}_{P}$ split over $\mathbb{Q}$, i.e., isomorphic to $\mathbb{C}^{\times}$over $\mathbb{Q}$, and let $A_{P}=\mathbf{S}_{P}(\mathbb{R})^{0}$, the identity component of the real locus $\mathbf{S}_{P}(\mathbb{R})$. Then there is a complementary subgroup $\mathbf{M}_{P}$ of $\mathbf{L}_{P}$ defined over $\mathbb{Q}$ such that $L_{P}=M_{P} A_{P} \simeq M_{P} \times A_{P}$, where $M_{P}=\mathbf{M}_{P}(\mathbb{R})$.

Under the lift $i_{0}: \mathbf{L}_{P} \rightarrow \mathbf{P}$, we identity $A_{P}, M_{P}$ with their images $i_{0}\left(A_{P}\right), i_{0}\left(M_{P}\right)$ in $P$. Then we have the following (rational) Langlands decomposition of $P$ :

$$
P=N_{P} M_{P} A_{P} \simeq N_{P} \times M_{P} \times A_{P},
$$

i.e., the map $(n, m, a) \rightarrow n m a$ is a diffeomorphism from $N_{P} \times A_{P} \times M_{P}$ to $P$. The dimension of $A_{P}$ is called the split rank of $\mathbf{P}$, and the $\mathbb{Q}$-rank of $\mathbf{G}$ is the maximum of the split rank of all rational parabolic subgroups $\mathbf{P}$ of $\mathbf{G}$.

When the $\mathbb{Q}$-rank of $\mathbf{G}$ is equal to $1, \Gamma \backslash X$ is called a $\mathbb{Q}$-rank 1 locally symmetric space. More generally, the $\mathbb{Q}$-rank of $\mathbf{G}$ is also called the $\mathbb{Q}$-rank of $\Gamma \backslash X$. For $n \geqslant 2$, the space $S L(n, \mathbb{Z}) \backslash S L(n, \mathbb{R}) / S O(n)$ has $\mathbb{Q}$-rank $n-1$, and hence is of $\mathbb{Q}$-rank 1 if and only if $n=2$. An important class $\Gamma \backslash X$ of $\mathbb{Q}$-rank 1 consists of Hilbert modular varieties. See [7] for details.

Since $G=P K$, the parabolic subgroup $P$ acts transitively on $X=G / K$, and the Langlands decomposition of $P$ induces the following horospherical decomposition of $X$ :

$$
X \cong N_{P} \times M_{P} /(K \cap P) \times A_{P},
$$

where the map is given by $(n, a, m(K \cap P), a) \rightarrow n a m K \in X$. Note that this map is well-defined since $A_{P}, M_{P}$ commute. In the above identification, we used the fact that $K \cap P=K \cap M_{P}$ is a maximal compact subgroup of $M_{P}$. Denote $K \cap M_{P}$ by $K_{P}$, and $M_{P} / K_{P}$ by $X_{P}$, called the boundary symmetric space associated with the rational parabolic subgroup $P$. Then the horospherical decomposition can be written as

$$
X=N_{P} \times X_{P} \times A_{P}
$$


This is a basic decomposition in this paper and plays an important role in describing the geometry at infinity of $\Gamma \backslash X$ and the continuous spectrum of $\Gamma \backslash X$. When $X=S L(2, \mathbb{R}) / S O(2)$ is identified with the upper half plane and $P$ consists of upper triangular matrices, $X_{P}$ reduces to a point, $N_{P}$ corresponds to the $x$-coordinates, and $A_{P}$ to the $y$-coordinates. For a general $\mathbb{Q}$-rank 1 space $\Gamma \backslash X, X_{P}$ is nontrivial. For example, for the Hilbert modular varieties in [7], $X_{P}$ is an Euclidean space.

In the following, the coordinates of a point $x \in X$ in this horospherical decomposition are denoted by

$$
x=(n(x), m(x), a(x)) \in N_{P} \times X_{P} \times A_{P} .
$$

Let $\mathfrak{a}_{P}$ be the Lie algebra of $A_{P}$, then $H(x)=\log a(x) \in \mathfrak{a}_{P}$, and $x$ is also written as

$$
x=\left(n(x), m(x), \mathrm{e}^{H(x)}\right) .
$$

The arithmetic subgroup $\Gamma$ induces several subgroups. Let $\Gamma_{N_{P}}=\Gamma \cap N_{P}$. Then $\Gamma_{N_{P}}$ is a cocompact discrete subgroup in $N_{P}$. Under the projection $P \rightarrow L_{P}$, the subgroup $\Gamma_{P}=\Gamma \cap P$ is mapped to an arithmetic subgroup of $\mathbf{L}_{P}$, which turns out to be contained in $\mathbf{M}_{P}$. Denote this image in $M_{P}$ by $\Gamma_{X_{P}}$ and identify it with its lift in $P$ under $i_{0}$. Then $\Gamma_{X_{P}}$ acts properly discontinuously on $X_{P}$ with a quotient of finite volume. In general, $\Gamma_{X_{P}}$ is not torsion free even if $\Gamma$ is. On the other hand, if we assume $\Gamma$ to be neat, i.e., every element in $\Gamma$ realized as a matrix via a linear embedding of $G$ has no root of unity except 1 as an eigenvalue, then $\Gamma$ is torsion free, and furthermore, $\Gamma_{X_{P}}$ is also torsion free. It is known that any arithmetic subgroup contains a neat subgroup of finite index. In fact, the neat condition is motivated by this property. (See [1, §17].) For convenience, we assume in the rest of the paper that $\Gamma$ is neat. The quotient $\Gamma_{X_{P}} \backslash X_{P}$ is a smooth locally symmetric space and called the boundary component of $\Gamma \backslash X$ associated with the rational parabolic subgroup $P$.

Let $\Phi^{+}\left(P, A_{P}\right)$ be the set of roots of the adjoint action of $A_{P}$ on $N_{P}$. For any real number $r$, define

$$
A_{P, r}=\left\{a \in A_{P} \mid \alpha(\log a)>r, \text { for all } \alpha \in \Phi^{+}\left(P, A_{P}\right)\right\},
$$

in particular, when $r=0, A_{P, r}$ is the positive chamber $A_{P}^{+}$. The corresponding chamber in $\mathfrak{a}_{P}$ is denoted by $\mathfrak{a}_{P}^{+}=\log A_{P}^{+}$. For any bounded set $w$ in $N_{P} \times X_{P}$, the set $w \times A_{P, r}$ in $N_{P} \times X_{P} \times A_{P} \simeq X$ is called a Siegel set of $X$ associated with the rational parabolic subgroup $P$.

The main result in the reduction theory of arithmetic subgroups can be summarized as follows (see [3],[23]).

PROPOSITION 2.2. - There are only finitely many $\Gamma$-conjugacy classes of (proper) rational parabolic subgroups of $G$. Let $P_{1}, \ldots, P_{n}$ be a set of representatives of these conjugacy classes. For every $r \gg 0$, and each $P_{i}$, there is a bounded set $w_{i}=w_{i}(r)$ such that $w_{i}$ is mapped injectively to a compact set in $\Gamma_{P} \backslash N_{P} \times X_{P}$ and $w_{i} \times A_{P_{i}, r}$ is injectively into $\Gamma \backslash X$ under the map $\pi: X=N_{P} \times X_{P} \times A_{P} \rightarrow \Gamma \backslash X$, and there is also a compact set $w_{0}=w_{0}(r)$ in $\Gamma \backslash X$ such that the following disjoint decomposition holds:

$$
\Gamma \backslash X=w_{0} \cup \coprod_{i=1}^{n} \pi\left(w_{i} \times A_{P_{i}, r}\right) .
$$

For convenience, we often identify $w_{i} \times A_{P_{i}, r}$ with its image $\pi\left(w_{i} \times A_{P_{i}, r}\right)$ in $\Gamma \backslash X$. Basically, the reduction theory says that the noncompactness of $\Gamma \backslash X$ comes from the Siegel sets of rational 
parabolic subgroups. For the case of a Riemann surface $\Gamma \backslash H$, each Siegel set $\pi\left(w_{i} \times A_{P_{i}, r}\right)$ corresponds to a cusp neighborhood of $\Gamma \backslash H$. So, in general, we can say that there is a one-toone correspondence between the "cusps" of $\Gamma \backslash X$ and $\Gamma$-conjugacy classes of rational parabolic subgroups of $\mathbf{G}$.

In the $\mathbb{Q}$-rank 1 case, the shape of $\Gamma \backslash X$ near infinity and the reduction theory can be described more explicitly as follows. In this case, all rational parabolic subgroups $P_{i}$ have split rank 1, i.e., $\operatorname{dim} A_{P_{i}}=1$, and $\Gamma_{X_{P_{i}}} \backslash X_{P_{i}}$ is a compact locally symmetric space. Since $\Gamma_{P_{i}}, \Gamma_{N_{P_{i}}}$ and $\Gamma_{X_{P_{i}}}$ fit into an exact sequence $0 \rightarrow \Gamma_{N_{P_{i}}} \rightarrow \Gamma_{P_{i}} \rightarrow \Gamma_{X_{P_{i}}} \rightarrow 0$, and $\Gamma_{N_{P_{i}}}$ is a normal subgroup of $\Gamma_{P_{i}}$, the quotient $\Gamma_{P_{i}} \backslash\left(N_{P_{i}} \times X_{P_{i}}\right)$ is a fiber bundle over $\Gamma_{X_{P_{i}}} \backslash X_{P_{i}}$ with fiber equal to $\Gamma_{N_{P_{i}}} \backslash N_{P_{i}}$, which is a compact nilmanifold. The bundle $\Gamma_{P_{i}} \backslash\left(N_{P_{i}} \times X_{P_{i}}\right)$ admits a flat connection whose (horizontal) sections are images of $\{n\} \times X_{P_{i}}$. Such sections will appear later as parameter spaces for scattering geodesics.

Proposition 2.3. - Assume $\mathbf{G}$ is of $\mathbb{Q}$-rank 1, i.e., the $\mathbb{Q}$-rank of $\Gamma \backslash X$ is equal to 1. When $r \gg 0$, two points in $N_{P_{i}} \times X_{P_{i}} \times A_{P_{i, r}} \subset X$ are $\Gamma$-equivalent if and only if they are $\Gamma_{P_{i}}$-equivalent, and hence $\Gamma_{P_{i}} \backslash N_{P_{i}} \times X_{P_{i}} \times A_{P_{i, r}}$ is mapped injectively into $\Gamma \backslash X$ under the projection $\Gamma_{P_{i}} \backslash X \rightarrow \Gamma \backslash X$. Each subset $\Gamma_{P_{i}} \backslash N_{P_{i}} \times X_{P_{i}} \times A_{P_{i, r}}$ is a topological cylinder with a section equal to $\Gamma_{P_{i}} \backslash N_{P_{i}} \times X_{P_{i}}$, and is an end of $\Gamma \backslash X$. Furthermore, all ends of $\Gamma \backslash X$ are of this form, and hence there exists a compact subset $w_{0} \subset \Gamma \backslash X$ such that $\Gamma \backslash X$ admits a disjoint decomposition:

$$
\Gamma \backslash X=w_{0} \cup \coprod_{i=1}^{n} \Gamma_{P_{i}} \backslash N_{P_{i}} \times X_{P_{i}} \times A_{P_{i, r}} .
$$

Briefly, the above proposition says that in the $\mathbb{Q}$-rank 1 case, all the ends of $\Gamma \backslash X$ are topological cylinders, with one end corresponding to one $\Gamma$-conjugacy class of rational parabolic subgroups. On the other hand, we would like to emphasize that each end is not a metric cylinder which is defined to be an isometric product $\mathbb{R}_{\geqslant 0} \times B$, where $B$ is a compact Riemannian manifold. In fact, in the decomposition

$$
\Gamma_{P_{i}} \backslash\left(N_{P_{i}} \times X_{P_{i}} \times A_{P_{i, r}}\right)=\left(\Gamma_{P_{i}} \backslash N_{P_{i}} \times X_{P_{i}}\right) \times A_{P_{i, r}},
$$

when the $A_{P_{i, r}}$-component goes to the positive infinity, the fibers $\Gamma_{N_{P_{i}}} \backslash N_{P_{i}}$ in the bundle $\Gamma_{P_{i}} \backslash N_{P_{i}} \times X_{P_{i}}$ shrink exponentially while the base $\Gamma_{X_{P_{i}}} \backslash X_{P_{i}}$ stays fixed. In a certain sense, an end of a $\mathbb{Q}$-rank 1 locally symmetric space $\Gamma \backslash X$ is combination of a cusp of a Riemann surface and a (metric) cylindrical end, where the shrinking factor $\Gamma_{N_{P_{i}}} \backslash N_{P_{i}}$ corresponds to the horocycle of a cusp, and $\Gamma_{X_{P_{i}}} \backslash X_{P_{i}}$ corresponds to a section of a cylindrical end. This combination will also be reflected in the description of the spectral decomposition of $\Gamma \backslash X$, in particular, the continuous spectrum.

Next we study geodesics in $\Gamma \backslash X$ which run from one "cusp" to another one, the so-called scattering geodesics.

Definition 2.4. - A geodesic $\gamma:(-\infty, \infty) \rightarrow \Gamma \backslash X$ is called a scattering geodesic if there exist two numbers $t_{1}<t_{2}$ and two rational parabolic subgroups $\mathbf{P}_{i}, \mathbf{P}_{j}$ such that (1) for all $t \geqslant t_{2}, \gamma(t) \in w_{i} \times A_{P_{i, r}}$ in Proposition 2.2 for some large $r$, and in the decomposition $\gamma(t)=$ $(n, m, a(t))$, the components $n, m$ are independent of $t$ for $t \geqslant t_{2}$, and $\log a(t)=t H+H_{0}$, where $H_{0} \in \mathfrak{a}_{P}, H \in \mathfrak{a}_{P}^{+}$. (2) The same conditions are satisfied by $\gamma(t)$ for $t \leqslant t_{1}$ with respect to the rational parabolic subgroup $\mathbf{P}_{j}$, and $\log a(t)=-t H+H_{0}$. (Note that the minus sign in front of $s$ is needed since $t \rightarrow-\infty$ in this case.) When the above conditions are satisfied, $\gamma$ is called a scattering geodesics between the ends associated with $P_{i}, P_{j}$, or a scattering geodesic from $P_{i}$ to $P_{j}$. 
In the $\mathbb{Q}$-rank 1 case, a scattering geodesic $\gamma$ runs from the infinity of one end to the infinity of another end, and inside each end, the coordinate on the section $\Gamma_{P_{i}} \backslash N_{P_{i}} \times X_{P_{i}}$ is constant. This latter condition is automatically satisfied for Riemann surfaces. On the other hand, in the general $\mathbb{Q}$-rank 1 case, there are geodesics going out to infinity of an end which do not satisfy this condition, i.e., the component of $\Gamma_{X_{P_{i}}} \backslash X_{P_{i}}$ is not constant and they spiral out to infinity. Therefore, in the general $\mathbb{Q}$-rank 1 case, scattering geodesics are not exactly the geodesics going from one end to another. But the additional condition imposed in the above definition is natural in view of the following result (see [16]).

Proposition 2.5. - A geodesic $\gamma$ in $\Gamma \backslash X$ is a scattering geodesic if and only if there exist two numbers $t_{1}<t_{2}$ such that both rays $\gamma(t), t \geqslant t_{2} ; \gamma(t), t \leqslant t_{1}$ are distance minimizing in $\Gamma \backslash X$, i.e., $\gamma$ is eventually distance minimizing in both directions.

More importantly, the condition on the scattering geodesics is also natural from the point of view of microlocal analysis, in particular, the wave equation as seen below. In the following, we only consider scattering geodesics between parabolic subgroups of rank 1 .

For each parabolic subgroup $\mathbf{P}$ of rank 1, i.e., $\operatorname{dim} A_{P}=1$, and for every sufficiently large height $r$, let $Y_{P, r}=\Gamma_{P} \backslash N_{P} \times X_{P} \times\{r\}$, a section at height $r$ of the end associated with $P$. Then $Y_{P, r}$ is a codimension 1 submanifold in $\Gamma \backslash X$. Let $N Y_{P, r}$ be the normal bundle of $Y_{P, r}$. The complement of the zero section in $N Y_{P, r}$ has two connected components. Denote the one containing the positive direction $A_{P}^{+}$by $N^{+} Y_{P, r}$, and the other component by $N^{-} Y_{P, r}$. Then we have the following characterization of scattering geodesics.

Proposition 2.6. - A geodesic $\gamma(t)$ is a scattering geodesic from a parabolic subgroup $\mathbf{P}_{i}$ to another parabolic subgroup $\mathbf{P}_{j}$ if and only if for every sufficiently large $r$, there exist $t_{1}, t_{2}$ such that $\gamma\left(t_{1}\right) \in Y_{P_{i}, r}, \gamma^{\prime}\left(t_{1}\right) \in N^{+} Y_{P_{i}, r}$, and $\gamma\left(t_{2}\right) \in Y_{P_{j}, r}, \gamma^{\prime}\left(t_{2}\right) \in N^{-} Y_{P_{j}, r}$.

Proof. - If $\gamma(t)$ is a scattering geodesic, it is clear that it satisfies the conditions in the proposition. On the other hand, if $\gamma(t)$ satisfies the above condition, then a lift $\tilde{\gamma}$ of $\gamma(t)$ in the universal covering space $X$ is of the form $\tilde{\gamma}(t)=\left(n_{i}, m_{i}, a_{i}(t)\right) \in N_{P_{i}} \times X_{P_{i}} \times A_{P_{i}}$ in the horospherical decomposition of $X$ with respect to $P_{i}$, where $n_{i}, m_{i}$ are independent of $t$, and $\log a_{i}(t)$ is linear in $t$. The reason for these expressions is that every geodesic in $X$ is of the form $\left(n, m, \exp \left(H_{0}+t H\right)\right) \in N_{P} \times X_{P} \times A_{P}$ for a unique real parabolic subgroup $P$ of $G$, where $n \in N_{P}, m \in X_{P}$, and $H \in \mathfrak{a}_{P}^{+}$. Since the $A_{P_{i}}$ component of $\tilde{\gamma}(t)$ goes to $+\infty$ when $t \rightarrow+\infty$, it is clear that $A_{P_{i}} \subset A_{P}$ and $P \subset P_{i}$. The conditions $\tilde{\gamma}\left(t_{1}\right) \in \widetilde{Y}_{P_{i}, r}=N_{P_{i}} \times X_{P_{i}} \times\{r\}$ and $\tilde{\gamma}^{\prime}\left(t_{1}\right) \in N^{+} \widetilde{Y}_{P_{i}, r}$ imply that $A_{P}=A_{P_{i}}$ and hence $P=P_{i}$. By considering the direction $t \rightarrow-\infty$, we get a similar expression for another lift $\tilde{\gamma}(t)$ in the horospherical decomposition determined by $P_{j}$. These expressions imply that $\gamma(t)$ is a scattering geodesic as defined in Definition 2.4.

An important invariant of a scattering geodesics is its sojourn time. In the following, all geodesics have unit speed and are directed.

Definition 2.7. - Let $r$ be a sufficiently large height, as in Proposition 2.2. For a scattering geodesic $\gamma(t)$ as in Definition 2.4, let $t_{2}(r)$ be the largest number $t$ such that $\gamma(t) \in Y_{P_{i}, r}$ the first time as $t$ decreases from $+\infty$. Similarly, let $t_{1}(r)$ be the smallest number $t$ such that $\gamma(t) \in Y_{P_{j}, r}$ for the first time as $t$ increases from $-\infty$. Then the sojourn time of $\gamma$ is defined to be $t_{2}(r)-t_{1}(r)-2 r$, and will be denoted by $T(\gamma)$.

We observe that $t_{2}\left(r^{\prime}\right)-t_{2}(r)=t_{1}(r)-t_{1}\left(r^{\prime}\right)=r-r^{\prime}$ and hence $t_{2}(r)-t_{1}(r)-2 r$ is independent of the height $r$. The sojourn times defined with this modification are not necessarily positive, though they are uniformly bounded from below by $-2 r$. 
In the case of Riemann surfaces, this sojourn time was first introduced by Guillemin [9]. In the $\mathbb{Q}$-rank 1 case, the sojourn time of a scattering geodesic is the length of the geodesic segment between two sections $Y_{P_{i}, r}, P_{P_{j}, r}$ of the ends of $P_{i}, P_{j}$ at the height $r$ modified by the height $r$ so that it is independent of $r$.

Proposition 2.8. - If $X$ has strictly negative sectional curvature, i.e., the rank of $X$ (or the $\mathbb{R}$-rank of $\mathbf{G})$ is equal to 1 , then there are countably infinitely many scattering geodesics between every pair of ends, and their sojourn times form a discrete sequence of numbers with finite multiplicity in $\mathbb{R}$.

Proof. - Two unit speed geodesics $\gamma(t), \gamma^{\prime}(t)$ in $X$ are defined to be equivalent if

$$
\lim _{t \rightarrow+\infty} \sup d\left(\gamma(t), \gamma^{\prime}(t)\right)<+\infty
$$

Let $X(\infty)$ be the set of equivalence classes of geodesics in $X$, called the sphere at infinity, and $X \cup X(\infty)$ the usual geodesic compactification. Since the rank of $X$ is equal to 1 , there is a one-to-one correspondence between real parabolic subgroups of $G$ and the points in $X(\infty)$, and furthermore, for any two distinct points $p, q$ in $X(\infty)$, there is a unique geodesic $\gamma(t)$ in $X$ such that $\lim _{t \rightarrow+\infty} \gamma(t)=p$, and $\lim _{t \rightarrow-\infty} \gamma(t)=q$. For each parabolic subgroup $P$, denote the corresponding point in $X(\infty)$ by $[P]$.

Fix two representatives $P_{i}, P_{j}$ of $\Gamma$-conjugacy classes of rational parabolic subgroups as in Proposition 2.2. For any two rational parabolic subgroups $P_{i}^{\prime}, P_{j}^{\prime}$ which are $\Gamma$-equivalent to $P_{i}, P_{j}$, let $\gamma(t)$ be the unique geodesic connecting the points $\left[P_{i}^{\prime}\right],\left[P_{j}^{\prime}\right]$ as above. Then the image of $\gamma$ in $\Gamma \backslash X$ is a scattering geodesic from the end of $P_{i}$ to the end of $P_{j}$. Conversely, any scattering geodesic from the end of $P_{i}$ to that of $P_{j}$ is of this form.

To show that the sojourn times form a discrete sequence, we denote the unique geodesic $\gamma(t)$ in $X$ connecting $\left[P_{i}^{\prime}\right],\left[P_{j}^{\prime}\right]$ by $\overline{P_{i}^{\prime}, P_{j}^{\prime}}$. Then every scattering geodesic connecting the ends of $P_{i}, P_{j}$ is the image in $\Gamma \backslash X$ of one of the geodesics in the union

$$
\bigcup_{\gamma \in \Gamma_{P_{i}} \backslash \Gamma} \overline{\gamma^{-1} P_{i} \gamma, P_{j}} .
$$

As $\gamma$ runs over the cosets and off to infinity, it can be shown that the sojourn time of the geodesic $\overline{\gamma^{-1} P_{i} \gamma, P_{j}}$ goes to infinity. The reason is that the sojourn time of $\overline{\gamma^{-1} P_{i} \gamma, P_{j}}$ is greater than the norm of the $\mathfrak{a}_{P_{i}}$-component of $\gamma$ in the Langlands decomposition, up to a constant independent of $\gamma$. By the proof of absolute convergence of Eisenstein series for large parameters (see [11, $\S 2])$, these norms form a discrete sequence. This implies that the sojourn times of the scattering geodesics between the ends of $P_{i}$ and $P_{j}$ form a discrete sequence. Since $\Gamma \backslash X$ has only finitely many ends, the discreteness of the spectrum of the sojourn times of $\Gamma \backslash X$ follows.

An immediate corollary of the above proposition is that if the $\mathbb{R}$-rank of $G$ is equal to 1 , the scattering geodesics of $\Gamma \backslash X$ are isolated. On the other hand, if the $\mathbb{R}$-rank of $G$ is greater than one, scattering geodesics are often embedded in smooth, non-discrete families.

Proposition 2.9. - Assume that the $\mathbb{Q}$-rank of $\mathbf{G}$ is equal to 1 . Let $\gamma(t)$ be a scattering geodesic in $\Gamma \backslash X$ between the ends associated with two rational parabolic subgroups $P_{i}$ and $P_{j}$. Then $\gamma(t)$ lies in a smooth family of scattering geodesics of the same sojourn time parametrized by a common finite covering space $X_{T, i j}$ of the boundary locally symmetric spaces $\Gamma_{X_{P_{i}}} \backslash X_{P_{i}}$ and $\Gamma_{X_{P_{j}}} \backslash X_{P_{j}}$.

Proof. - First, we assume that a lift $\tilde{\gamma}(t)$ in $X$ of $\gamma(t)$ is of the form $\tilde{\gamma}(t)=(i d, m, a(t)) \in$ $N_{P_{i}} \times X_{P_{i}} \times A_{P_{i, r}}$, where $m \in X_{P_{i}}, a(t)=\exp H_{0}+t H, H \in \mathfrak{a}_{P_{i}}^{+}$. Let $P_{i}^{-}$be the opposite 
parabolic subgroup of $P_{i}$ with respect to the split component $A_{P_{i}}$, i.e., $P_{i}^{-}=N_{P_{i}}^{-} M_{P_{i}} A_{P_{i}}$, where the Lie algebra of $N_{P_{i}}^{-}$is equal to sum of the root spaces of the roots $-\alpha$, for $\alpha \in \Phi^{+}\left(P, A_{P_{i}}\right)$. Since the $N_{P_{i}}$ component of $\tilde{\gamma}(t)$ is trivial, the horospherical coordinates of $\tilde{\gamma}(t)$ for $P_{i}$ are also the horospherical coordinates for $P_{i}^{-}$. Therefore, $\tilde{\gamma}(t)$ is a scattering geodesic between $P_{i}$ and $P_{i}^{-}$. By assumption, $\tilde{\gamma}(t)$ is a scattering geodesic between $P_{i}$ and a $\Gamma$-conjugate of $P_{j}$. This implies that $P_{i}^{-}$is a rational parabolic subgroup, hence the lifted Levi quotient $i_{0}\left(M_{P_{i}} A_{P_{i}}\right)$, which is also denoted by $M_{P_{i}} A_{P_{i}}$, is given by $M_{P_{i}} A_{P_{i}}=P_{i} \cap P_{i}^{-}$and hence is a rational subgroup. In particular, the lifted subgroup $M_{P_{i}}=i_{x_{0}}\left(M_{P_{i}}\right)$ is defined over $\mathbb{Q}$, and the split component $A_{P_{i}}$ is also rational.

The above argument shows that in the equation $\tilde{\gamma}(t)=(i d, m, a(t))$, the component $m$ can be changed to another point in $X_{P_{i}}$ and $\tilde{\gamma}(t)$ is still a scattering geodesic between $P_{i}$ and $P_{i}^{-}$. It is clear that the sojourn time of $\tilde{\gamma}(t)$ does not depend on $m$. The projections of two such geodesics $\tilde{\gamma}_{1}(t)=\left(i d, m_{1}, a(t)\right), \tilde{\gamma}_{2}(t)=\left(i d, m_{2}, a(t)\right)$ in $\Gamma \backslash X$ define the same geodesic if and only if there exists an element $\gamma \in \Gamma$ such that $\gamma m_{1}=m_{2}$. This implies that $\gamma \in M_{P_{i}}$ and hence $\gamma \in \Gamma \cap M_{P_{i}}=\Gamma_{P_{i}} \cap M_{P_{i}}$. Therefore, the projection $\gamma(t)$ of $\tilde{\gamma}(t)=(i d, m, a(t))$ in $\Gamma \backslash X$ only depends on the image of $m$ in the quotient $\Gamma_{P_{i}} \cap M_{P_{i}} \backslash X_{P_{i}}$, and hence the scattering geodesic $\gamma(t)$ belongs to a smooth family parametrized by $\Gamma_{P_{i}} \cap M_{P_{i}} \backslash X_{P_{i}}$. The space $\Gamma_{P_{i}} \cap M_{P_{i}} \backslash X_{P_{i}}$ can be identified with the image of $\{i d\} \times X_{P_{i}}$ in the bundle $\Gamma_{P_{i}} \backslash N_{P_{i}} \times X_{P_{i}}$ and hence is a horizontal section of this bundle (see the comments before Proposition 2.3). This implies that $\Gamma_{P_{i}} \cap M_{P_{i}} \backslash X_{P_{i}}$ is a covering space of the base $\Gamma_{X_{P_{i}}} \backslash X_{P_{i}}$ of this bundle. Since $\Gamma$ is torsion free, $\Gamma_{P_{i}} \cap M_{P_{i}}$ is also torsion free, and hence $\Gamma_{P_{i}} \cap M_{P_{i}} \backslash X_{P_{i}}$ is a smooth manifold which we will denote by $X_{T, i j}$.

As mentioned earlier, $M_{P_{i}}$ is a rational subgroup of $P_{i}$. This implies that $\Gamma_{P_{i}} \cap M_{P_{i}}$ is an arithmetic subgroup of $M_{P_{i}}$ and hence a subgroup of finite index of $\Gamma_{X_{P_{i}}}$, which is defined earlier as the image of $\Gamma_{P_{i}}$ in $M_{P_{i}}$ under the projection defined by the Langlands decomposition. Therefore, $\Gamma_{P_{i}} \cap M_{P_{i}} \backslash X_{P_{i}}$ is a finite covering space of $\Gamma_{X_{P_{i}}} \backslash X_{P_{i}}$. Since $P_{j}$ is $\Gamma$-conjugate to $P_{i}^{-}$and $M_{P_{i}^{-}}=M_{P_{i}}$, it follows that $X_{P_{j}}=X_{P_{i}}$ and $\Gamma_{P_{j}} \cap M_{P_{j}}=\Gamma \cap M_{P_{j}}=\Gamma_{P_{i}} \cap M_{P_{i}}$, and $\Gamma_{P_{j}} \cap M_{P_{j}} \backslash X_{P_{j}}$ can be identified with $\Gamma_{P_{i}} \cap M_{P_{i}} \backslash X_{P_{i}}$. This implies that $\Gamma_{P_{i}} \cap M_{P_{i}} \backslash X_{P_{i}}$ is a finite common covering space of $\Gamma_{X_{P_{i}}} \backslash X_{P_{i}}$ and $\Gamma_{X_{P_{j}}} \backslash X_{P_{j}}$. The proposition is proved in this case.

On the other hand, suppose that a lift $\tilde{\gamma}(t)$ has a non-trivial $N_{P_{i}}$-component $n, \tilde{\gamma}(t)=$ $(n, m, a(t))$. Let $x_{1}=n x_{0}$ be a new basepoint. Then with respect to this basepoint $x_{1}$, the Langlands decomposition of $P_{i}$ becomes

$$
P_{i}=N_{P_{i}} \times\left(n M_{P_{i}} n^{-1}\right) \times\left(n A_{P_{i}} n^{-1}\right) .
$$

In the induced horospherical decomposition of $X$ :

$$
X=N_{P_{i}} \times n M_{P_{i}} n^{-1} /\left(n K_{P_{i}} n^{-1}\right) \times n A_{P_{i}} n^{-1},
$$

the components of $\tilde{\gamma}(t)$ are given by

$$
\tilde{\gamma}(t)=\left(i d, n m n^{-1}\left(n K_{P_{i}} n^{-1}\right), n a(t) n^{-1}\right) .
$$

Since the $N_{P_{i}}$-component is trivial, by the previous argument, $\tilde{\gamma}(t)$ is a scattering geodesic between $P_{i}$ and opposite parabolic subgroup of $P_{i}$ with respect to this split component $n A_{P_{i}} n^{-1}$. If we denote the opposite parabolic subgroup of $P_{i}$ with respect to the fixed split component $A_{P_{i}}$ as above by $P_{i}^{-}$, then this opposite parabolic subgroup with respect to the split component $n A_{P_{i}} n^{-1}$ is equal to $n P_{i}^{-} n^{-1}$. By assumption, $\gamma(t)$ is a scattering geodesic between two 
rational parabolic subgroups $P_{i}$ and $P_{j}$. This implies that $n P_{i}^{-} n^{-1}$ is also rational and $\Gamma$ conjugate to $P_{j}$. This in turn implies that the split component $n A_{P_{i}} n^{-1}$ is rational, and the lift $i_{x_{1}}\left(M_{P_{i}}\right)=n M_{P_{i}} n^{-1}$ is also defined over $\mathbb{Q}$.

As in the previous case, the $M_{P_{i}}$ component of $\tilde{\gamma}(t)$ can be moved in $X_{P_{i}}$ and $\tilde{\gamma}(t)$ is still a scattering geodesic of the same sojourn time. Two such geodesics $\left(n, m_{1}, a(t)\right)$ and $\left(n, m_{2}, a(s)\right)$ in $X$ project to the same geodesic in $\Gamma \backslash X$ if and only if there exists an element $\gamma \in \Gamma$ such that $\gamma n m_{1}=n m_{2}$. This implies that $n^{-1} \gamma n \in n^{-1} \Gamma n \cap M_{P_{i}}$. Therefore, the image in $\Gamma \backslash X$ of a geodesic $(n, m, a(t))$ in $X$ only depends on the image of $m$ in $n^{-1} \Gamma n \cap M_{P_{i}} \backslash X_{P_{i}}$. This shows that $\gamma(t)$ belongs to a smooth family of scattering geodesics parametrized by $n^{-1} \Gamma n \cap M_{P_{i}} \backslash X_{P_{i}}$. As above, since $\Gamma$ is torsion free, $n^{-1} \Gamma n \cap M_{P_{i}}$ is also torsion free and hence the parameter space $n^{-1} \Gamma n \cap M_{P_{i}} \backslash X_{P_{i}}$ is a smooth manifold. This parameter space can be identified with the image of the horizontal section $\{n\} \times X_{P_{i}}$ in the bundle $\Gamma_{P_{i}} \backslash N_{P_{i}} \times X_{P_{i}}$ and hence is a covering space of the base $\Gamma_{X_{P_{i}}} \backslash X_{P_{i}}$. In fact it is a finite covering space of $\Gamma_{X_{P_{i}}} \backslash X_{P_{i}}$. First note that $n^{-1} \Gamma n \cap M_{P_{i}} \backslash X_{P_{i}}$ can be identified with $\Gamma \cap n M_{P_{i}} n^{-1} \backslash n M_{P_{i}} n^{-1} /\left(n K_{P_{i}} n^{-1}\right)$. Since $n M_{P_{i}} n^{-1}$ is a rational subgroup, the intersection $\Gamma \cap n M_{P_{i}} n^{-1}$ is an arithmetic subgroup in $n M_{P_{i}} n^{-1}$, and hence $n^{-1} \Gamma n \cap M_{P_{i}}$ is a cofinite discrete subgroup of $M_{P_{i}}$. This proves the finiteness of the covering $n^{-1} \Gamma n \cap M_{P_{i}} \backslash X_{P_{i}} \rightarrow \Gamma_{X_{P_{i}}} \backslash X_{P_{i}}$. The corresponding smooth manifold will be denoted by $X_{T, i j}$.

Since $P_{j}$ is $\Gamma$-conjugate to $n P_{i}^{-} n^{-1}$ and $n^{-1} \Gamma n \cap M_{P_{i}} \backslash X_{P_{i}} \cong \Gamma \cap n M_{P_{i}} n^{-1} \backslash X_{P_{i}}$ can be identified with $\Gamma \cap M_{P_{j}} \backslash X_{P_{j}}$, it follows that the parameter space $n^{-1} \Gamma n \cap M_{P_{i}} \backslash X_{P_{i}}$ is also a common finite covering space of $\Gamma_{X_{P_{i}}} \backslash X_{P_{i}}$ and $\Gamma_{X_{P_{j}}} \backslash X_{P_{j}}$. This completes the proof of this proposition.

Remark. - If a sojourn time has a multiplicity, then we will have different manifolds $X_{T, i j}$ for each different family of geodesics with the same sojourn time. To fix an identification of $X_{T, i j}$, we will identify it with the quotient of $X_{P_{i}}$ as in the proof of the proposition.

Remark. - In this paper, we fixed a basepoint $x_{0}=K \in X$ and identified the subgroup $M_{P}$ in the Levi quotient of a rational parabolic subgroup $P$ with its lift $i_{x_{0}}\left(M_{P}\right)$. In general, $i_{x_{0}}\left(M_{P}\right)$ is not a rational subgroup. But for any rational parabolic subgroup $P$, we can always find a basepoint $x_{1}$ such that $i_{x_{1}}\left(M_{P}\right)$ is rational. The existence of such a basepoint can be seen from the proof of the above proposition.

Suppose that $i_{x_{0}}\left(M_{P}\right)$ is rational. Then the set of connected components of the continuous families of scattering geodesics coming out of the parabolic subgroup $P$ are parametrized by $\Gamma_{N_{P}} \backslash N_{P}(\mathbb{Q})$. Precisely, let $m_{0}=K_{P}$ be a basepoint in $X_{P}=M_{P} / K_{P}$. Let $H$ be the unique unit vector in $\mathfrak{a}_{P}^{+}$. Then the proof of the above proposition shows that for every $n \in \mathbf{N}_{P}(\mathbb{Q})$, the geodesic $\left(n, m_{0}, \exp t H\right)$ in $X$ projects to a scattering geodesic in $\Gamma \backslash X$ coming from $P$. Two such scattering geodesics belong to one connected family if and only if their $N_{P}$ components are in the same $\Gamma_{N_{P}}$ orbit. Furthermore, every scattering geodesic coming out of $P$ is of this form.

This parametrization of scattering geodesics coming out of a cusp can be seen clearly in the case of Riemann surfaces.

Each connected family of scattering geodesics between two rational parabolic subgroups or two ends of $\Gamma \backslash X$ has a common sojourn time. All these sojourn times form a spectrum of sojourn times. In the following, we say a geodesic in $X$ is a scattering geodesic if its image in $\Gamma \backslash X$ is a scattering geodesic, and its sojourn time is equal to the sojourn time of the image.

Proposition 2.10. - Assume that $\mathbb{Q}$-rank of $\Gamma \backslash X$ is equal to 1 as above. Then for any two (not necessarily different) ends of $\Gamma \backslash X$, there are countably infinitely many smooth families of scattering geodesics between them, and the spectrum of sojourn times of all scattering geodesics forms a discrete sequence of points in $\mathbb{R}$ of finite multiplicities. 
Proof. - We first prove that for any two different rational parabolic subgroups $P_{1}, P_{2}$ of $G$, there is a unique connected family of scattering geodesics in $X$ which project to a family of scattering geodesics in $\Gamma \backslash X$ of the same sojourn time. By [5, Proposition 4.7], $P_{1}, P_{2}$ contains a unique common maximal rational split torus $A: A \subset P_{1} \cap P_{2}$. Since $\mathbf{G}$ has $\mathbb{Q}$ rank $1, A$ is a common split component of $P_{1}, P_{2}$. Let $P_{1}=N_{P_{1}} M_{P_{1}} A, P_{2}=N_{P_{2}} M_{P_{2}} A$ be their Langlands decomposition. Then $M_{P_{1}}=M_{P_{2}}$. Since $P_{1}$ is different from $P_{2}, N_{P_{1}} \neq N_{P_{2}}$, and $P_{2}$ is the opposite parabolic subgroup of $P_{1}$ with respect to the split component $A$. Then the proof of Proposition 2.9 shows that there is a smooth family of scattering geodesics in $X$ between $P_{1}$ and $P_{2}$. Its projection in $\Gamma \backslash X$ gives a smooth family of scattering geodesics of the same sojourn time parametrized by $\Gamma \cap M_{P_{1}} \backslash X_{P_{1}}$.

Let $P_{1}, \ldots, P_{n}$ be representatives of $\Gamma$-conjugacy classes of rational parabolic subgroups, which corresponds to the set of ends of $\Gamma \backslash X$ as mentioned earlier. Suppose two ends of $\Gamma \backslash X$ correspond to $P_{i}$ and $P_{j}$. When $P_{i}=P_{j}$, i.e., when the two ends agree, for any $\gamma \in \Gamma \backslash \Gamma_{P_{i}}$, $P_{i} \neq \gamma P_{i} \gamma^{-1}$. Then the family of scattering geodesics in $X$ between $P_{i}$ and $\gamma P_{i} \gamma^{-1}$ projects to a family of scattering geodesics in $\Gamma \backslash X$ between this common end. Conversely, every scattering geodesic in $\Gamma \backslash X$ between this end lies in such a family. This shows that there are countably infinitely many families of scattering geodesics connecting any one end. On the other hand, when $P_{i} \neq P_{j}, P_{i} \neq \gamma P_{j} \gamma^{-1}$ for every $\gamma \in \Gamma$, and the family between $P_{i}$ and $\gamma P_{j} \gamma^{-1}$ projects to a family of scattering geodesics in $\Gamma \backslash X$ between the two ends, and any family of scattering geodesics in $\Gamma \backslash X$ between the two ends is of this form. This shows that for any two different ends, there are countably infinitely many families of scattering geodesics between them. This proves the first part of the proposition.

For every pair of parabolic subgroups $P_{i}, P_{j}$ as above and $\gamma \in \Gamma$, when $P_{i} \neq \gamma P_{j} \gamma^{-1}$, the sojourn time of the family of scattering geodesics determined by $P_{i}$ and $\gamma P_{j} \gamma^{-1}$ is greater than the norm of the $\mathfrak{a}_{P_{j}}$-component of $\gamma$ in the Langlands decomposition with respect to $P_{j}$, up to a constant independent of $\gamma$. By the proof of absolute convergence of Eisenstein series for large parameters (see [11, 2 , Remark 1]), these norms form a discrete sequence in $\mathbb{R}$. This in turn implies the discreteness of the spectrum of the sojourn times of all the scattering geodesics in $\Gamma \backslash X$.

Remark. - When the $\mathbb{Q}$-rank of $\Gamma \backslash X$ is equal to 1 , it is easy to see directly that there are scattering geodesics between any two different ends. In fact, for each end, pick a sequence of points in $\Gamma \backslash X$ converging the infinity of that end and connect these two sequences of points by distance minimizing geodesic segments. Then any limit of such a sequence of geodesic segments is a required scattering geodesic. But this argument does not work if two ends are the same.

Let $T(\Gamma \backslash X)$ be the tangent bundle of $\Gamma \backslash X$, and $S(\Gamma \backslash X)$ be the unit sphere bundle.

Proposition 2.11. - Assume that the $\mathbb{Q}$-rank of $\Gamma \backslash X$ is equal to 1 . Let $P_{1}, \ldots, P_{n}$ be representatives of $\Gamma$-conjugacy classes of rational parabolic subgroups. Let $\Phi^{t}$ be the geodesic flow in the tangent bundle $T(\Gamma \backslash X)$ minus the zero section. For every pair $P_{i}, P_{j}$, let $Y_{P_{1}, r}$, $Y_{P_{2}, r}$ be the sections at the height $r \gg 0$ (defined before Proposition 2.6). Then $\Phi^{T}\left(N Y_{P_{i}, r}\right) \cap$ $N Y_{P_{j}, r} \neq \emptyset$ if and only if one of the following holds:

(1) $T=0$, and $P_{i}=P_{j}$ and hence $Y_{P_{i}, r}=Y_{P_{j}, r}$.

(2) $T \neq 0$, and $|T|-2 r$ is the sojourn time of a family of scattering geodesic between the ends associated with $P_{i}$ and $P_{j}$, and each connected component of the family is a common finite cover of $\Gamma_{X_{P_{i}}} \backslash X_{P_{i}}$ and $\Gamma_{X_{P_{j}}} \backslash X_{P_{j}}$.

If $\Phi^{T}\left(N Y_{P_{i}, r}\right)$ and $N Y_{P_{j}, r}$ intersect, they intersect cleanly, and the excess is equal to $\operatorname{dim} X_{T, i j}=\operatorname{dim} X_{P_{i}}=\operatorname{dim} X_{P_{j}}$. (In this proposition and below, the zero sections of the normal 
bundles $N Y_{P_{i}, r}, N Y_{P_{j}, r}$ have been removed.) Furthermore, $\Phi^{T}\left(N Y_{P_{i}, r}\right) \cap N Y_{P_{j}, r} \neq \emptyset$ if and only if $\Phi^{-T}\left(N Y_{P_{i}, r}\right) \cap N Y_{P_{j}, r} \neq \emptyset$.

Proof. - When $r \gg 0$, Proposition 2.3 shows that $Y_{P_{i}, r}$ is disjoint from $Y_{P_{j}, r}$ when $P_{i} \neq P_{j}$. This implies that when $T=0, \Phi^{T}\left(N Y_{P_{i}, r}\right) \cap N Y_{P_{j}, r} \neq \emptyset$ if and only if $P_{i}=P_{j}$.

Next we consider the case $T \neq 0$. Since $\Phi^{T}\left(N Y_{P_{i}, r}\right) \cap N Y_{P_{j}, r} \neq \emptyset$ if and only if $N Y_{P_{i}, r} \cap$ $\Phi^{-T}\left(N Y_{P_{j}, r}\right) \neq \emptyset$. We can assume $T>0$. Let $N^{ \pm} Y_{P, r}$ be the two connected components of the complement in $N Y_{P, R}$ of the zero section as in Proposition 2.6. When $r \gg 0, \Phi^{T}\left(N^{+} Y_{P_{i}, r}\right)=$ $N^{+} Y_{P_{i}, r+T}$. By Proposition 2.3 again, $Y_{P_{i}, r+T}$ is disjoint from $Y_{P_{j}, r}$. This implies that

$$
\Phi^{T}\left(N Y_{P_{i}, r}\right) \cap N Y_{P_{j}, r}=\Phi^{T}\left(N^{-} Y_{P_{i}, r}\right) \cap N^{+} Y_{P_{j}, r} .
$$

Let $S(\Gamma \backslash X)$ be the unit sphere bundle in the tangent bundle $T(\Gamma \backslash X)$. Since the geodesic flow preserves the length, $\Phi^{T}\left(N^{-} Y_{P_{i}, r}\right) \cap N^{+} Y_{P_{j}, r} \cap S(\Gamma \backslash X) \neq \emptyset$. By Proposition 2.6, the orbit under the geodesic flow $\Phi^{t}$ of any intersection point in

$$
\Phi^{T}\left(N^{-} Y_{P_{i}, r}\right) \cap N^{+} Y_{P_{j}, r} \cap S(\Gamma \backslash X)
$$

is a scattering geodesic from $P_{j}$ and $P_{i}$, and $|T|-2 r$ is exactly the sojourn time of this scattering geodesic. Each connected component of this intersection set $\Phi^{T}\left(N^{-} Y_{P_{i}, r}\right) \cap N^{+} Y_{P_{j}, r} \cap$ $S(\Gamma \backslash X)$ parametrizes a continuous family of scattering geodesics between $P_{i}$ and $P_{j}$ and hence is a common finite cover of $\Gamma_{X_{P_{i}}} \backslash X_{P_{i}}$ and $\Gamma_{X_{P_{j}}} \backslash X_{P_{j}}$ by Proposition 2.9. This proves the first statement.

We next prove the clean intersection property. Recall that two submanifolds $M_{1}, M_{2}$ of a manifold $M$ intersect cleanly if $T M_{1} \cap T M_{2}=T\left(M_{1} \cap M_{2}\right)$. Clearly, when $T=0$ and $P_{i}=P_{j}$, $\Phi^{T}\left(N Y_{P_{i}, r}\right)$ intersects $N Y_{P_{j}, r}$ cleanly. As above, we can assume now that $T>0$. Since each connected component of $\Phi^{T}\left(N Y_{P_{i}, r}\right) \cap N Y_{P_{j}, r}$ corresponds to a continuous family of scattering geodesics which has a common sojourn time, the previous proposition then implies that there are at most only finitely connected components of the intersection. Each connected component can be studied via its lift in $T X$. Let $\widetilde{Y}_{P, r}$ be the lift in $X$ of $Y_{P, r}$, and $N \widetilde{Y}_{P, r}$ the normal bundle of $\widetilde{Y}_{P, r}$ in $T X$. The intersection of $\Phi^{T}\left(N Y_{P_{i}, r}\right)$ and $N Y_{P_{j}, r}$ is clean if the intersection of their lifts $\Phi^{T}\left(N \widetilde{Y}_{P_{i}, r}\right) \cap N \widetilde{Y}_{\gamma P_{j} \gamma^{-1}, r}$ in $T X$ is clean for all $\gamma \in \Gamma$. The reason is that discreteness of $\Gamma$ shows that locally we can identify the quotient with the lift, so if that is done for all lifts associated with $\gamma \in \Gamma$, we have cleanness of the intersection in the quotient.

For simplicity of notation, we only prove the case when $\gamma=i d$. Under the geodesic flow $\Phi^{t}$, $\Phi^{T}\left(N^{+} \widetilde{Y}_{P_{i}, r}\right)=N^{+} \widetilde{Y}_{P_{i}, r+T}$, and $\Phi^{T}\left(N^{-} \widetilde{Y}_{P_{i}, r}\right)=N^{-} \widetilde{Y}_{P_{i}, r-T}$. Then as observed earlier, for $T \geqslant 0$

$$
\Phi^{T}\left(N \widetilde{Y}_{P_{i}, r}\right) \cap N \widetilde{Y}_{P_{j}, r}=\Phi^{T}\left(N^{-} \widetilde{Y}_{P_{i}, r}\right) \cap N^{+} \widetilde{Y}_{P_{j}, r} .
$$

Let $v$ be a point in $\Phi^{T}\left(N^{-} \widetilde{Y}_{P_{i}, r}\right) \cap N^{+} \widetilde{Y}_{P_{j}, r} \cap S(\Gamma \backslash X)$. The $G$-orbit of $v$ in $T X$ is a smooth submanifold which is diffeomorphic to $G / K_{P_{i}}$, since the stabilizer of $v$ in $G$ is equal to $K_{P_{i}}$. The orbit of $v$ under the subgroup $N_{P_{i}} M_{P_{i}}$ is equal to $N^{-} \widetilde{Y}_{P_{i}, r} \cap S(\Gamma \backslash X)$, which is diffeomorphic to $N_{P_{i}} \times X_{P_{i}}$. And the map from $N_{P_{i}} M_{P_{i}}$ to its orbit $N^{-} \widetilde{Y}_{P_{i}, r} \cap S(\Gamma \backslash X)$ in $G \cdot v$ is a submersion. The same conclusion holds for $P_{j}$. As explained in the proof of Propositions 2.9 and 2.11, $P_{i}$ and $P_{j}$ are the opposite parabolic subgroups of a common split component, and hence for their Langlands decompositions with respect to this common split component, $M_{P_{i}}=M_{P_{j}}, N_{P_{j}}=N_{P_{i}-}$. Then the sets $\Phi^{T}\left(N^{-} \widetilde{Y}_{P_{i}, r}\right) \cap S(\Gamma \backslash X), N^{+} \widetilde{Y}_{P_{j}, r} \cap S(\Gamma \backslash X)$ can be identified with the orbits of the common submanifold $M_{P_{i}} \cong \cong X_{P_{i}}$ in $G \cdot v \subset T X$ under the groups $N_{P_{i}}, N_{P_{j}}$, and the intersection set $\Phi^{T}\left(N^{-} \widetilde{Y}_{P_{i}, r}\right) \cap N^{+} \widetilde{Y}_{P_{j}, r} \cap S(\Gamma \backslash X)$ is the common 
submanifold $M_{P_{i}} v \cong X_{P_{i}}$. The normal directions of $M_{P_{i}} v \cong X_{P_{i}}$ in $\Phi^{T}\left(N^{-} \widetilde{Y}_{P_{i}, r}\right) \cap S(\Gamma \backslash X)$ and $N^{+} \widetilde{Y}_{P_{j}, r} \cap S(\Gamma \backslash X)$ can be identified with the Lie algebras $\mathfrak{n}_{P_{i}}, \mathfrak{n}_{P_{j}}$ of $N_{P_{i}}, N_{P_{j}}=N_{P_{i}}{ }^{-}$ respectively when the $G$-orbit through $v$ is identified with $G / K_{P_{i}}$. Since these Lie algebras $\mathfrak{n}_{P_{i}}, \mathfrak{n}_{P_{j}}=\mathfrak{n}_{P_{i}}^{-1}$ are sums of root spaces of roots of different signs, $\mathfrak{n}_{P_{i}} \cap \mathfrak{n}_{P_{j}}=\{0\}$, and hence $\Phi^{T}\left(N^{-} \widetilde{Y}_{P_{i}, r}\right) \cap S(\Gamma \backslash X)$ and $N^{+} \widetilde{Y}_{P_{j}, r} \cap S(\Gamma \backslash X)$ intersect cleanly at $M_{P_{i}} v \cong X_{P_{i}}$. By scaling the length of the normal vectors, this implies that $\Phi^{T}\left(N^{-} \widetilde{Y}_{P_{i}, r}\right)$ and $N^{+} \widetilde{Y}_{P_{j}, r}$ also intersect cleanly. Furthermore, the excess, $\operatorname{codim}\left(T\left(\Phi^{T}\left(N^{-} \widetilde{Y}_{P_{i}, r}\right)\right)+\left(T N^{+} \widetilde{Y}_{P_{j}, r}\right)\right)$, is equal to $\operatorname{dim} X_{i}+\operatorname{dim} A_{i}=\operatorname{dim} X_{i}+1$. This follows from the decomposition $X=N_{P_{i}} \times A_{P_{i}} \times X_{P_{i}}$ and the fact that $\mathfrak{n}_{P_{i}} \cap \mathfrak{n}_{P_{j}}=\{0\}$, $\operatorname{dim} \mathfrak{n}_{P_{i}}=\operatorname{dim} \mathfrak{n}_{P_{j}}$.

To prove the last statement, assume $T>0$. We observe as above that

$$
\begin{gathered}
\Phi^{T}\left(N^{-} Y_{P_{i}, r}\right) \cap N^{+} Y_{P_{j}, r}=\Phi^{T}\left(N Y_{P_{i}, r}\right) \cap N Y_{P_{j}, r}, \\
\Phi^{-T}\left(N^{+} Y_{P_{i}, r}\right) \cap N^{-} Y_{P_{j}, r}=\Phi^{-T}\left(N Y_{P_{i}, r}\right) \cap N Y_{P_{j}, r} .
\end{gathered}
$$

Since $\Phi^{T}\left(N^{-} Y_{P_{i}, r}\right) \cap N^{+} Y_{P_{j}, r} \neq \emptyset$ if and only if $\Phi^{-T}\left(N^{+} Y_{P_{i}, r}\right) \cap N^{-} Y_{P_{j}, r} \neq \emptyset$, the last statement is clear.

Remark. - Let $S X$ be the unit sphere bundle in $T X$. Then $G$ acts on $S X$. This $G$-action is transitive if and only if the rank of $X$, i.e., the $\mathbb{R}$-rank of $G$ is equal to 1 , and the action is simply transitive if and only if $G=S L(2, \mathbb{R})$. In this latter case, $S X$ can be identified with $G$. When the rank of $X$ is greater than 1, there are infinitely many $G$-orbits in $S X$, and each of them is a smooth submanifold.

Remark. - For higher rank rational parabolic subgroups $P$, i.e., when $\operatorname{dim} A_{P}>1$, scattering geodesics between them also lie in continuous families. In fact each smooth family of scattering geodesics between $P_{i}$ and $P_{j}$ is parametrized by the product of a common finite cover of $\Gamma_{X_{P_{i}}} \backslash X_{P_{i}}$ and $\Gamma_{X_{P_{j}}} \backslash X_{P_{j}}$, and a vector subspace contained in $\mathfrak{a}_{P_{i}}$, which is the orthogonal complement of the $\mathfrak{a}_{P_{i}}$ component of the geodesics. In the $\mathbb{Q}$-rank 1 case, $\operatorname{dim} \mathfrak{a}_{P}=1$, and the factor of the vector subspace reduces to a point. In the higher rank case, it should be the scattering flats, i.e., flat subspaces which are immersions of $\mathbb{R}^{l} \cong \mathfrak{a}_{P}$, that play the role of scattering geodesics in the $\mathbb{Q}$-rank 1 case. It can be shown that each smooth family of scattering flats between $P_{i}, P_{j}$ is parametrized by a common finite cover of $\Gamma_{X_{P_{i}}} \backslash X_{P_{i}}$ and $\Gamma_{X_{P_{j}}} \backslash X_{P_{j}}$.

Let $T^{*}(\Gamma \backslash X)$ be the cotangent bundle of $\Gamma \backslash X, S^{*}(\Gamma \backslash X)$ the unit sphere bundle in $S^{*}(\Gamma \backslash X)$, $N^{*} Y_{P, r}$ the conormal bundle. Under the Riemannian metric, these co-bundles can be identified with the corresponding bundles. Then the results of this section are summarized in Theorem 1 and in the following commutative diagram: when $T \neq 0$,

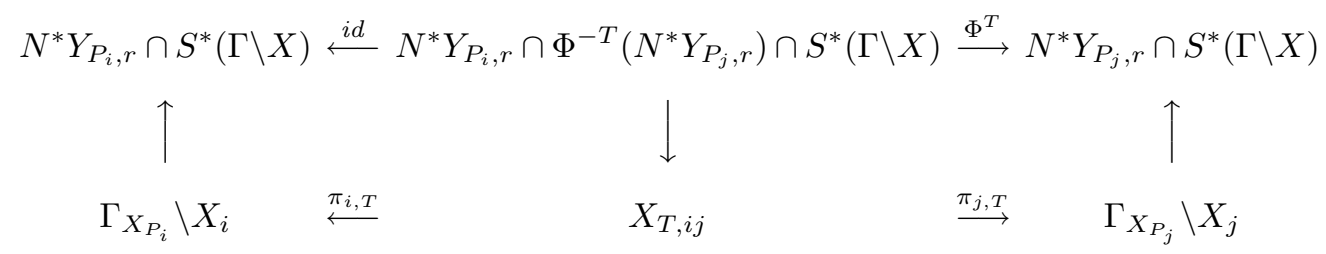

The space $X_{T, i j}$ parametrizes the family of scattering geodesics between the ends of $P_{i}$ and $P_{j}$ with sojourn time $|T|-2 r$, and because of the clean intersection property (Proposition 2.11) it can be identified with $N^{*} Y_{P_{i}, r} \cap \Phi^{-T}\left(N^{*} Y_{P_{j}, r}\right) \cap S^{*}(\Gamma \backslash X)$ : the middle vertical map is a diffeomorphism. 


\section{Spectral theory of locally symmetric spaces}

In this section, we recall the spectral decomposition for locally symmetric spaces $\Gamma \backslash X$, and a decomposition suitable for studying the relation between the sojourn times of scattering geodesics and the scattering matrices in the continuous spectrum. For example, the latter decomposition shows that for a $\mathbb{Q}$-rank 1 space $\Gamma \backslash X, L^{2}(\Gamma \backslash X)$ can be thought of as a countable sum of $L^{2}(S)$ where $S$ is a Riemann surface, and these summands do not interact with each other for the purpose of scattering theory. Under compact perturbations, such a decomposition does not hold in general. This is the reason that the results in Theorem 2 do not hold for compact perturbations of $\Gamma \backslash X$ as mentioned in the introduction. In the following, we always identify a function on $\Gamma \backslash X$ with its lift on $X$, which is a $\Gamma$-invariant function on $X$.

For any locally integrable function $f$ on $\Gamma \backslash X$, and any rational parabolic subgroup $P$, the constant term $f_{P}$ of $f$ along $P$ is defined by

$$
f_{P}(x)=\int_{\Gamma_{N_{P}} \backslash N_{P}} f(n x) \mathrm{d} n,
$$

where the Haar measure $\mathrm{d} n$ on $\Gamma_{N_{P}} \backslash N_{P}$ has total measure 1. Clearly $f_{P}$ is invariant under $N_{P}$ and hence $f_{P}$ is a function on $\Gamma_{X_{P}} \backslash X_{P} \times A_{P}$.

A function $\varphi$ on $\Gamma \backslash X$ is called cuspidal if for all proper rational parabolic subgroups $P$ of $G$, the constant terms $f_{P}$ vanish. The subspace of $L^{2}(\Gamma \backslash X)$ consisting of cuspidal functions is called the cuspidal subspace and denoted by $L_{\text {cus }}^{2}(\Gamma \backslash X)$. Let $\Delta$ be the Beltrami-Laplace operator of $\Gamma \backslash X$. Then an important result of Gelfand and Piatetski-Shapiro [8] is the following

PROPOSITION 3.1. - The restriction of $\Delta$ to $L_{\text {cus }}^{2}(\Gamma \backslash X)$ has a discrete spectrum.

Let $L_{\text {dis }}^{2}(\Gamma \backslash X)$ be the subspace of $L^{2}(\Gamma \backslash X)$ spanned by all $L^{2}$-eigenfunctions of $\Delta$, and $L_{\text {con }}^{2}(\Gamma \backslash X)$ the orthogonal complement of $L_{\text {dis }}^{2}(\Gamma \backslash X)$ in $L^{2}(\Gamma \backslash X)$. Then $L_{\text {cus }}^{2}(\Gamma \backslash X)$ is contained in $L_{\text {dis }}^{2}(\Gamma \backslash X)$ and the inclusion is proper. For example, the constant functions are in $L_{\text {dis }}^{2}(\Gamma \backslash X)$ but not in $L_{\text {cus }}^{2}(\Gamma \backslash X)$. Both the continuous subspace $L_{\text {con }}^{2}(\Gamma \backslash X)$ and the noncuspidal discrete spectrum $L_{\text {dis }}^{2}(\Gamma \backslash X)-L_{\text {cus }}^{2}(\Gamma \backslash X)$ can be described in terms of Eisenstein series. In fact, $L_{\text {dis }}^{2}(\Gamma \backslash X)-L_{\text {cus }}^{2}(\Gamma \backslash X)$ is spanned by square integrable residues of (linear combinations of) Eisenstein series, and hence is called the residue subspace, denoted by $L_{\text {res }}^{2}(\Gamma \backslash X)$.

For any proper rational parabolic subgroup $P$, let $\rho_{P}$ be half the sum of roots in $\Phi^{+}\left(P, A_{P}\right)$ with the multiplicity equal to the dimension of the root spaces. For any $x \in X$, write the horospherical coordinates as above:

$$
x=\left(n(x), m(x), \mathrm{e}^{H(x)}\right) \in N_{P} \times X_{P} \times A_{P}=X,
$$

where $H(x) \in \mathfrak{a}_{P}$. For any cuspidal eigenfunction $\phi$ on the boundary locally symmetric space $\Gamma_{X_{P}} \backslash X_{P}$, and $\Lambda \in \mathfrak{a}_{P}^{*} \otimes \mathbb{C}$ with $\operatorname{Re} \Lambda \gg 0$ in the sense that $\alpha(\operatorname{Re} \Lambda) \gg 0$ for all $\alpha \in \Phi^{+}\left(P, A_{P}\right)$, the Eisenstein series

$$
E(P, \phi, \Lambda)(x)=\sum_{\Gamma_{P} \backslash \Gamma} \mathrm{e}^{\left(\rho_{P}+\Lambda\right)(H(\gamma x))} \phi(m(\gamma x))
$$

converge absolutely and uniformly for $x$ in compact subsets of $X$. Clearly, $E(P, \phi, \Lambda)$ is $\Gamma$-invariant and hence defines a function on $\Gamma \backslash X$. The Eisenstein series $E(P, \phi, \Lambda)$ is locally integrable on $\Gamma \backslash X$ but does not belong to $L^{2}(\Gamma \backslash X)$. 
Remark. - When $X$ is the upper half plane, the above definition reduces to the usual definition. In this case, $\phi=1$ or constant, $\rho_{P}=\frac{1}{2}$, and $H(z)=\log \operatorname{Im}(z), z \in X=\{z \in \mathbb{C} \mid \operatorname{Im}(z)>0\}$.

To get functions in $L^{2}(\Gamma \backslash X)$, we need to define pseudo-Eisenstein series (or incomplete theta series). For any $v \in C_{0}^{\infty}\left(\mathfrak{a}_{P}\right)$, define

$$
E(P, \phi, v)(x)=\sum_{\Gamma_{P} \backslash \Gamma} v(H(\gamma x)) \phi(m(\gamma x)) .
$$

This series converges absolutely and uniformly for $x$ in compact subsets in $X$, and $E(P, \phi, v) \in$ $L^{2}(\Gamma \backslash X)$. We note that formally, if we take $v(H)=\exp \left(\rho_{P}+\Lambda\right)(H)$, the pseudo-Eisenstein series becomes the Eisenstein series, and hence the pseudo-Eisenstein series are truncated versions of the Eisenstein series.

For a fixed $P$ and a cuspidal eigenfunction $\phi$ on $\Gamma_{X_{P}} \backslash X_{P}$, when $v$ runs over all functions in $C_{0}^{\infty}\left(\mathfrak{a}_{P}\right)$, the pseudo-Eisenstein series $E(P, \phi, v)$ span a subspace of $L^{2}(\Gamma \backslash X)$ whose closure is invariant under $\Delta$. Denote this closed subspace by $L_{P, \phi}^{2}(\Gamma \backslash X)$. Then it is a basic fact due to Langlands ([18], see also [11, p. 16]) that the subspaces $L_{P, \phi}^{2}(\Gamma \backslash X)$ for all possible choices of the pair $P, \phi$ span the orthogonal complement of $L_{\text {cus }}^{2}(\Gamma \backslash X)$ in $L^{2}(\Gamma \backslash X)$, in other words,

$$
L^{2}(\Gamma \backslash X)=L_{\text {cus }}^{2}(\Gamma \backslash X) \oplus \sum_{P, \phi} L_{P, \phi}^{2}(\Gamma \backslash X),
$$

and hence

$$
L_{\text {con }}^{2}(\Gamma \backslash X) \oplus L_{\text {res }}^{2}(\Gamma \backslash X)=\sum_{P, \phi} L_{P, \phi}^{2}(\Gamma \backslash X) .
$$

But a difficulty with the right hand side is that the subspaces $L_{P, \phi}^{2}(\Gamma \backslash X)$ are not orthogonal to each other and hence the sum is not a direct sum. Therefore, we need to understand the interaction (or intersection) of these subspaces. The scattering matrices introduced below describe this interaction and overcome the difficulty.

DEFINITION 3.2. - Two rational parabolic subgroups $P_{1}, P_{2}$ are called associate if there exists an element $g \in G$ such that $g A_{P_{1}} g^{-1}=A_{P_{2}}$.

Remark. - In the above definition, $A_{P_{1}}, A_{P_{2}}$ are lifts in $G$ of the identity components of the split center of the Levi quotients $\mathbf{L}_{P_{1}}, \mathbf{L}_{P_{2}}$, and hence they are not necessarily defined over $\mathbb{Q}$ in general. On the other hand, if they are defined over $\mathbb{Q}$, then we can choose $g \in \mathbf{G}(\mathbb{Q})$ such that $g A_{P_{1}} g^{-1}=A_{P_{2}}$ (see [11, pp. 33-34]).

If the $\mathbb{Q}$-rank of $G$ is equal to 1 , then all parabolic subgroups are minimal and hence conjugate under $\mathbf{G}(\mathbb{Q})$ and hence associate.

LEMMA 3.3. - let $P_{1}, \phi_{1} ; P_{2}, \phi_{2}$ be two pairs as above. Then $L_{P_{1}, \phi_{1}}^{2}(\Gamma \backslash X)$ is perpendicular to $L_{P_{2}, \phi_{2}}^{2}(\Gamma \backslash X)$ if $P_{1}, P_{2}$ are not associate, or $P_{1}, P_{2}$ are associated but $\phi_{1}, \phi_{2}$ have different eigenvalues.

This lemma suggests the following decomposition of $L^{2}(\Gamma \backslash X)$. Since any two conjugate rational parabolic subgroups are automatically associate, there are finitely many associate classes of rational parabolic subgroups, denoted by $\mathcal{C}_{1}, \ldots, \mathcal{C}_{m}$. For each associate class $\mathcal{C}_{i}$, let $P_{i, 1}, \ldots, P_{i, r_{i}}$ be representatives of $\Gamma$-conjugacy classes in $\mathcal{C}_{i}$. Let $\operatorname{Spec}_{\text {cus }}\left(\mathcal{C}_{i}\right)$ be the union of 
the cuspidal eigenvalues of $\Gamma_{X_{P_{i, j}}} \backslash X_{P_{i, j}}, 1 \leqslant j \leqslant r_{r}$. Then for any $\mu \in \operatorname{Spec}_{\text {cus }}\left(\mathcal{C}_{i}\right)$, define

$$
L_{\mathcal{C}_{i, \mu}}^{2}(\Gamma \backslash X)=\sum L_{P_{i, j ; \phi}}^{2}(\Gamma \backslash X)
$$

where $1 \leqslant j \leqslant r_{i}$, and $\phi$ ranges over all cuspidal eigenfunctions $\phi$ on $\Gamma_{X_{P_{i, j}}} \backslash X_{P_{i, j}}$ with eigenvalue $\mu$. It is important to note that this sum is not direct.

Then the above discussions and Lemma 3.3 can be summarized into the following:

LEMmA 3.4. - For $1 \leqslant i \leqslant m, \mu \in \operatorname{Spec}_{\text {cus }}\left(\mathcal{C}_{i}\right), L^{2}(\Gamma \backslash X)$ admits the orthogonal decomposition

$$
L^{2}(\Gamma \backslash X)=L_{\text {cus }}^{2}(\Gamma \backslash X) \oplus \sum_{i, \mu} \oplus L_{\mathcal{C}_{i}, \mu}^{2}(\Gamma \backslash X),
$$

and this decomposition is invariant under $\Delta$.

As mentioned earlier, there is only one association class $\mathcal{C}$ in the $\mathbb{Q}$-rank 1 case. For simplicity, in this case, $L_{\mathcal{C}, \mu}^{2}(\Gamma \backslash X)$ is denoted by $L_{\mu}^{2}(\Gamma \backslash X)$ also, and then $L^{2}(\Gamma \backslash X)$ admits the following decomposition

$$
L^{2}(\Gamma \backslash X)=L_{\text {cus }}^{2}(\Gamma \backslash X) \oplus \sum_{\mu \in \operatorname{Spec}(\mathcal{C})} \oplus L_{\mu}^{2}(\Gamma \backslash X) .
$$

Besides being orthogonal, this decomposition in Lemma 3.4 has another property which is crucial for our study of the relation between the scattering matrices and the scattering geodesics. The property is that for any two distinct subspaces $L_{\mathcal{C}_{i, \mu}}^{2}(\Gamma \backslash X), L_{\mathcal{C}_{j, \nu}}^{2}(\Gamma \backslash X)$ and any two functions $f, g$ belonging to them, and for any rational parabolic subgroup $P$, the constant terms $f_{P}, g_{P}$ of $f, g$ along $P$ are perpendicular to each other if restricted to sections $\Gamma_{X_{P}} \backslash X_{P}$ in $\Gamma_{X_{P}} \backslash X_{P} \times A_{P}$. (Recall that this latter space is the space where the constant terms live.)

To get the spectral decomposition of each subspace $L_{\mathcal{C}_{i, \mu}}^{2}(\Gamma \backslash X)$ and hence $L^{2}(\Gamma \backslash X)$, we need meromorphic continuation of Eisenstein series $E(P, \phi, \Lambda)$, their constant terms, and functional equations satisfied by them.

Recall that $E(P, \phi, \Lambda)$ converges absolutely when $\operatorname{Re} \Lambda \gg 0$. Langlands [18] showed that $E(P, \phi, \Lambda)$ admits a meromorphic continuation in $\mathfrak{a}_{P}^{*} \otimes \mathbb{C}$.

LEMMA 3.5. - Let $P^{\prime}$ be a rational parabolic subgroup. If $\operatorname{rank}\left(P^{\prime}\right) \geqslant \operatorname{rank}(P)$ and $P^{\prime}$ is not associate to $P$, then the constant term $E_{P^{\prime}}(P, \phi, \Lambda)=0$. On the other hand, if $P^{\prime}$ is associate to $P$, then the constant term of $E(P, \phi, \Lambda)$ along $P^{\prime}$ is given by

$$
E_{P^{\prime}}(P, \phi, \Lambda)\left(m \mathrm{e}^{H}\right)=\sum_{W\left(\mathfrak{a}_{P}, \mathfrak{a}_{P^{\prime}}\right)} \mathrm{e}^{\left(\rho_{P^{\prime}}+s \Lambda\right)(H)}\left(c_{\mu}(s: \Lambda) \phi\right)(m),
$$

where $W\left(\mathfrak{a}_{P}, \mathfrak{a}_{P^{\prime}}\right)$ is the set of all linear maps from $\mathfrak{a}_{P}$ to $\mathfrak{a}_{P^{\prime}}$ of the form $\left.\operatorname{Ad}(g)\right|_{\mathfrak{a}_{P}}$, where $g$ satisfies $g A_{P} g^{-1}=A_{P^{\prime}}$ in the definition of associate parabolic subgroups, $\mu$ is the eigenvalue of the cuspidal eigenfunction $\phi$ and $c_{\mu}(s: \Lambda)$ is a meromorphic family of linear maps from the cuspidal eigenspace of $\Gamma_{X_{P}} \backslash X_{P}$ to the cuspidal eigenspace of $\Gamma_{X_{P^{\prime}}} \backslash X_{P^{\prime}}$ of the same eigenvalue $\mu$.

The linear maps $c_{\mu}(s: \Lambda)$ are called scattering matrices from $P$ to $P^{\prime}$ for the eigenvalue $\mu$. When $\operatorname{Re} \Lambda \gg 0$, this lemma is proved in [11, Chap. II, §4, 5]. By meromorphic continuation, the same results hold for all $\Lambda \in \mathfrak{a}_{P}^{*} \otimes \mathbb{C}$. 
Remark. - It is not obvious that a cuspidal eigenfunction $\phi$ on the boundary locally symmetric space $\Gamma_{X_{P}} \backslash X_{P}$ of eigenvalue $\mu$ is scattered only to another cuspidal eigenfunction on $\Gamma_{X_{P^{\prime}}} \backslash X_{P^{\prime}}$ of the same eigenvalue $\mu$. This fact is not clear from the eigen-equation $\Delta \phi=\mu \phi$ alone. In fact, we need all the $G$-invariant differential operators to draw this conclusion.

Since all the invariant differential operators of $X_{P}$ leaves the $\mu$-eigenspace in $L_{\text {cus }}^{2}\left(\Gamma_{X_{P}} \backslash X_{P}\right)$ invariant and commute with each other, we can assume that $\phi$ is a joint eigenfunction of all the invariant differential operators. Then $E(P, \phi, \Lambda)$ is also a joint eigenfunction of all the invariant differential operators of $X$, since each summand in the series is so. This implies the constant term $E_{P^{\prime}}(P, \phi, \Lambda)$ is also a joint eigenfunction with the same eigenvalue, which in turn implies that for each fixed $a$ in $A_{P^{\prime}}$, the restriction of $E_{P^{\prime}}(P, \phi, \Lambda)$ to $\Gamma_{X_{P^{\prime}}} \backslash X_{P^{\prime}} \times\{a\}$ in $\Gamma_{X_{P^{\prime}}} \backslash X_{P^{\prime}} \times A_{P^{\prime}}$ is a joint eigenfunction of all invariant differential operators on $X_{P^{\prime}}$, and in particular the eigenvalue for the Beltrami-Laplace operator is equal to $\mu$. In this brief argument, a crucial point is that the eigenvalues of a joint eigenfunction of all invariant differential operators on $X$ is determined by a point in a Cartan subalgebra up to the action of the Weyl group. This is the reason that $W\left(\mathfrak{a}_{P}, \mathfrak{a}_{p^{\prime}}\right)$ enters into the equation for $E_{P^{\prime}}(P, \phi, \Lambda)$ in the above lemma. For details, see [11, Chap. II, §5].

When $\operatorname{Re} \Lambda=0, E(P, \phi, \Lambda)$ are the generalized eigenfunctions for the continuous spectrum. These generalized eigenfunctions are not linearly independent. Instead, they are related by functional equations.

For simplicity, we assume in the rest of this section that $\mathbf{G}$ is of $\mathbb{Q}$-rank 1 unless otherwise specified, and state the functional equation for the Eisenstein series and the spectral decomposition in this $\mathbb{Q}$-rank 1 case. Near the end, we will comment on the higher rank case.

For every rational parabolic subgroup $P, \operatorname{dim} A_{P}=1$. Identify $\mathfrak{a}_{P}$ with $\mathbb{R}$ such that the norm on $\mathfrak{a}_{P}$ defined by the Killing form becomes the standard Euclidean norm of $\mathbb{R}$ and the roots in $\Phi^{+}\left(P, A_{P}\right)$ are positive linear functionals on $\mathbb{R}$. Similarly, we identify $\mathfrak{a}_{P}$ with $\mathbb{R}$, and hence $\mathfrak{a}_{P}^{*} \otimes \mathbb{C}$ with $\mathbb{C}$. Since $\Gamma_{X_{P}} \backslash X_{P}$ is compact, the cuspidal condition for functions on $\Gamma_{X_{P}} \backslash X_{P}$ is empty and hence automatically satisfied.

As mentioned earlier, there is only one association class $\mathcal{C}$ of parabolic subgroups, and $\Gamma_{X_{P}} \backslash X_{P}$ is compact for every rational parabolic subgroup $P$, and hence $\operatorname{Spec}_{\text {cus }}(\mathcal{C})=\operatorname{Spec}(\mathcal{C})$ is the union of the eigenvalues of the compact locally symmetric spaces $\Gamma_{X_{P_{i}}} \backslash X_{P_{i}}$, where $P_{1}, \ldots, P_{n}$ are representatives of $\Gamma$-conjugacy classes of rational parabolic subgroups. For every $\mu \in \operatorname{Spec}(\mathcal{C})$, and every $1 \leqslant j \leqslant n$, choose an orthonormal basis of the $\mu$-eigenspace of $\Gamma_{X_{P_{j}}} \backslash X_{P_{j}}$. As explained above, we can also choose them to be joint eigenfunctions of all the invariant differential operators on $X_{P_{j}}$. Put all these eigenfunctions into a list $\phi_{1}^{\mu}, \ldots, \phi_{K(\mu)}^{\mu}$. Each $\phi_{k}^{\mu}$ is associated with a unique rational parabolic subgroup $P_{j(k)}$ such that $\phi_{k}^{\mu}$ is an eigenfunction on $\Gamma_{X_{P_{j(k)}}} \backslash X_{P_{j(k)}}$, and hence defines an Eisenstein series $E\left(P_{j(k)}, \phi_{k}^{\mu}, \Lambda\right)$. For simplicity, we also denote the Eisenstein series by $E\left(\phi_{k}^{\mu}, \Lambda\right)$.

For an Eisenstein series $E\left(\phi_{k}^{\mu}, \Lambda\right)$ and a parabolic subgroup $P_{j}$, the constant term along $P_{j}$

$$
E_{P_{j}}\left(\phi_{k}^{\mu}, \Lambda\right)\left(m \mathrm{e}^{H}\right)=\delta_{j, j(k)} \mathrm{e}^{(\rho+\Lambda)(H)} \phi_{k}^{\mu}(m)+\sum_{i=1}^{K(\mu)} \mathrm{e}^{(\rho-\Lambda)(H)} c_{k i}(\Lambda) \phi_{i}^{\mu}(m),
$$

where $\rho=\rho_{P_{j}}$ is the half sum of roots in $\Phi^{+}\left(P_{j}, A_{P_{j}}\right)$ and is identified with a number in $\mathbb{R}$ as mentioned earlier, $\delta_{j, j(k)}$ is equal to 1 if $j=j(k)$ and zero otherwise, and the sum over $i$ runs over those $\phi_{i}^{\mu}$ which are eigenfunctions of the boundary space $\Gamma_{X_{P_{j}}} \backslash X_{P_{j}}$, and $c_{k i}(\Lambda)$ are components of the scattering matrix $c_{\mu}(s: \Lambda)$ in Lemma 3.5 with respect to the orthonormal basis $\phi_{1}^{\mu}, \ldots, \phi_{K(\mu)}^{\mu}$. 
For each $\mu \in \operatorname{Spec}(\mathcal{C})$, let $\mathcal{E}\left(\Gamma_{X_{j}} \backslash X_{P_{j}}, \mu\right)$ be the eigenspace of eigenvalue $\mu$, and

$$
\mathcal{E}(\mathcal{C}, \mu)=\bigoplus_{j=1}^{n} \mathcal{E}\left(\Gamma_{X_{j}} \backslash X_{P_{j}}, \mu\right)
$$

For any $\phi=\left(\psi_{1}, \ldots, \psi_{n}\right) \in \mathcal{E}(\mathcal{C}, \mu)$, define its Eisenstein series

$$
E(\phi, s)=\sum_{j=1}^{n} E\left(P_{j}, \psi_{j}, \Lambda\right) .
$$

The eigenfunctions $\phi_{1}^{\mu}, \ldots, \phi_{K(\mu)}^{\mu}$ can be identified with functions in $\mathcal{E}(\mathcal{C}, \mu)$ and form an orthonormal basis. With respect to this basis, the matrix $c_{\mu}(\Lambda)=\left(c_{i j}(\Lambda)\right)$ defines a linear map

$$
c_{\mu}(\Lambda): \mathcal{E}(\mathcal{C}, \mu) \rightarrow \mathcal{E}(\mathcal{C}, \mu)
$$

This matrix is called the scattering matrix for $\Gamma \backslash X$ at the eigenvalue $\mu$.

Then the functional equation for the Eisenstein series can be stated as follows: For any $\phi \in \mathcal{E}(\mathcal{C}, \mu)$,

$$
E\left(c_{\mu}(\Lambda) \phi,-\Lambda\right)=E(\phi, \Lambda) .
$$

From this equation, we obtain that

$$
c_{\mu}(\Lambda) c_{\mu}(-\Lambda)=I d
$$

Since $c_{\mu}(\Lambda)$ is unitary when $\operatorname{Re}(\Lambda)=0$, we get that when $\operatorname{Re}(\Lambda)=0$, the matrix $c_{\mu}(\Lambda)$ is also symmetric.

We note that the functional equation for Eisenstein series of a Riemann surface is stated in a different way that the scattering matrix acts on the Eisenstein series instead of the function inside, and the index for the scattering matrix is parametrized by the cusps. In the general $\mathbb{Q}$-rank 1 case, there are many Eisenstein series associated with one parabolic subgroup, and the functional equation (3.5) relates one Eisenstein series to a linear combination of Eisenstein series for functions $\psi_{j}$ defined on different boundary components $\Gamma_{X_{P_{i}}} \backslash X_{P_{i}}$ as defined in equation (3.4).

The Eisenstein series $E\left(\phi_{1}^{\mu}, \Lambda\right), \ldots, E\left(\phi_{K(\mu)}^{\mu}, \Lambda\right)$ have only finitely many poles for $\Lambda \in[0, \rho]$, and these poles give rise to the residual subspace $L_{\text {res }}^{2}(\Gamma \backslash X) \cap L_{\mu}^{2}(\Gamma \backslash X)$ which is therefore finite dimensional.

Using the above notation, we can describe the spectral decomposition of the subspace $L_{\mu}^{2}(\Gamma \backslash X)$ of $L^{2}(\Gamma \backslash X)$ (see Eq. (3.1)) in the following two propositions:

Proposition 3.6. - For every $\mu \in \operatorname{Spec}(\mathcal{C})=\bigcup_{i=1}^{n} \operatorname{Spec}\left(\Gamma_{P_{i}} \backslash X_{P_{i}}\right)$, let $e_{1}^{\mu}, \ldots, e_{L(\mu)}^{\mu}$ be an orthonormal basis of the finite dimensional residual subspace in $L_{\mu}^{2}(\Gamma \backslash X)$. Then for any $f \in L_{\mu}^{2}(\Gamma \backslash X)$, the following expression holds in the $L^{2}$-sense:

$$
f=\sum_{l=1}^{L(\mu)}\left(f, e_{l}^{\mu}\right) e_{l}^{\mu}+\frac{1}{4 \pi} \sum_{k=1}^{K(\mu)} \int_{\mathbb{R}}\left(f, E\left(\phi_{k}^{\mu}, \mathrm{i} \lambda\right)\right) E\left(\phi_{k}^{\mu}, \mathrm{i} \lambda\right) \mathrm{d} \lambda .
$$

PROPOSITION 3.7. - If $\mu_{1} \neq \mu_{2}$, then for any functions $f \in L_{\mu_{1}}^{2}(\Gamma \backslash X), g \in L_{\mu_{2}}^{2}(\Gamma \backslash X)$, and any rational parabolic subgroup $P$, the constant terms $f_{P}, g_{P}$ are orthogonal to each other when 
restricted to every section $\Gamma_{X_{P}} \backslash X_{P}$ in $\Gamma_{X_{P}} \backslash X_{P} \times A_{P}$, i.e., for any a $\in A_{P}$ :

$$
\int_{\Gamma_{X_{P}} \backslash X_{P}} f_{P}(m a) \overline{g_{P}}(m a) \mathrm{d} m=0 .
$$

Proof. - For two different eigenvalues $\mu_{1}, \mu_{2}$, the constant terms of their Eisenstein series $E\left(\phi_{i}^{\mu_{1}}, \Lambda\right)$ and $E\left(\phi_{j}^{\mu_{1}}, \Lambda\right)$ along any rational parabolic subgroup $P$ are given by eigenfunctions of different eigenvalues and hence orthogonal to each other when restricted to any section $\Gamma_{X_{P}} \backslash X_{P}$ in $\Gamma_{X_{P}} \backslash X_{P} \times A_{P}$. Clearly, the same conclusion holds for residual eigenfunctions. This proves the proposition.

This spectral decomposition of each subspace $L_{\mu}^{2}(\Gamma \backslash X)$ is similar to the spectral decomposition of a Riemann surface with $K(\mu)$ cusps. When the $\mathbb{Q}$-rank of $\Gamma \backslash X$ is equal to 1 , by (2.2),

$$
L^{2}(\Gamma \backslash X)=L_{\text {cus }}^{2}(\Gamma \backslash X) \oplus \sum_{\mu \in \operatorname{Spec}(\mathcal{C})} \oplus L_{\mu}^{2}(\Gamma \backslash X) .
$$

Putting together the spectral decomposition of $L_{\mu}^{2}(\Gamma \backslash X)$ for all $\mu \in \operatorname{Spec}(\mathcal{C})$ and $L_{\text {cus }}^{2}(\Gamma \backslash X)$, we get the spectral decomposition of $L^{2}(\Gamma \backslash X)$. Therefore, as commented in the beginning of this section, for $\mathbb{Q}$-rank 1 space $\Gamma \backslash X$, its spectral decomposition is similar to union of countably many Riemann surfaces. A basic reason why we need this decomposition is that even though the full residual space $L_{\text {res }}^{2}(\Gamma \backslash X)$ can be of infinite dimension, the residual subspace in each $L_{\mathcal{C}_{i}, \mu}^{2}(\Gamma \backslash X)$ is at most finite dimensional.

Both the functional equation for Eisenstein series and the spectral decomposition in the higher rank is much more complicated. Instead of giving detailed, complicated statements, we make several remarks.

Remark. - If the $\mathbb{Q}$-rank of $\mathbf{G}$ is greater than 1 , there are in general more than one association classes of rational parabolic subgroups of split rank 1 . For each such association class $\mathcal{C}_{i}$, the subspace $L_{\mathcal{C}_{i}, \mu}^{2}(\Gamma \backslash X)$ admits a spectral decomposition similar to the $\mathbb{Q}$-rank 1 case.

On the other hand, if the parabolic subgroups $P$ in an association class $\mathcal{C}_{i}$ have split rank greater than one, then the spectral decomposition of the subspace $L_{\mathcal{C}_{i}, \mu}^{2}(\Gamma \backslash X)$ is different in the sense that some new non-cuspidal Eisenstein series of parabolic subgroups not contained in the association class $\mathcal{C}_{i}$ arise, i.e., Eisenstein series $E(Q, \phi, \Lambda)$ associated with a non-cuspidal eigenfunction $\phi$ on $\Gamma_{X_{Q}} \backslash X_{Q}$, where $Q$ is a rational parabolic subgroup containing a subgroup $P$ in $\mathcal{C}_{i}$.

Remark. - Though the scattering matrices for higher rank rational parabolic subgroups are more complicated, they can be factorized into products of rank one scattering matrices of some boundary locally symmetric spaces. More precisely, let $\mathcal{C}_{i}$ be an association class of rank greater than or equal to 2 , then a scattering matrix for $\mathcal{C}_{i}$ is product of scattering matrices of parabolic subgroups in $\mathcal{C}_{i}$ when they are considered as rank 1 parabolic subgroups of larger parabolic subgroups. For details, see [11, pp. 124-125], [21, pp. 524-525], and [15, § 2.7]. From this point of view, it is crucial to understand the rank 1 scattering matrices.

\section{Scattering matrix in terms of the wave group}

In the $\mathbb{Q}$-rank one case, all the rational parabolic subgroups $\mathbf{P}$ are conjugate and hence the half sums $\rho_{P}$ of roots in $\Phi^{+}\left(P, A_{P}\right)$ are all equal if each is identified with a positive number as in the introduction. Denote this common number by $\rho$. 
For any $\mu \in \operatorname{Spec}(\mathcal{C})=\bigcup_{i=1}^{n} \operatorname{Spec}\left(\Gamma_{P_{i}} \backslash X_{P_{i}}\right)$, let $U^{\mu}(t)$ denote the following modified propagator for the wave equation acting on the subspace $L_{\mu}^{2}(\Gamma \backslash X)$ :

$$
U^{\mu}(t)=\cos t \sqrt{\Delta-\rho^{2}-\mu^{2}},
$$

where we choose the branch of the square root which is positive on the real axis and apply the spectral theorem. Using Proposition 3.6 we can write the wave group in terms of Eisenstein series: for $f \in L_{\mu}^{2}(\Gamma \backslash X)$,

$$
U^{\mu}(t) f=\sum_{l=1}^{L(\mu)} \cos \left(t \sqrt{\lambda_{l}^{\mu}-\rho^{2}-\mu^{2}}\right)\left(f, e_{l}^{\mu}\right) e_{l}^{\mu}+\frac{1}{4 \pi} \sum_{k=1}^{K(\mu)} \int_{\mathbb{R}}\left(f, E\left(\phi_{k}^{\mu}, \mathrm{i} \lambda\right)\right) E\left(\phi_{k}^{\mu}, \mathrm{i} \lambda\right) \mathrm{e}^{\mathrm{i} t \lambda} \mathrm{d} \lambda .
$$

In fact, by Proposition 3.6,

$$
\begin{aligned}
U^{\mu}(t) f= & \sum_{l=1}^{L(\mu)} \cos \left(t \sqrt{\lambda_{l}^{\mu}-\rho^{2}-\mu^{2}}\right)\left(f, e_{l}^{\mu}\right) e_{l}^{\mu} \\
& +\frac{1}{4 \pi} \sum_{k=1}^{K(\mu)} \int_{\mathbb{R}}\left(f, E\left(\phi_{k}^{\mu}, \mathrm{i} \lambda\right)\right) E\left(\phi_{k}^{\mu}, \mathrm{i} \lambda\right) \frac{1}{2}\left(\mathrm{e}^{\mathrm{i} t|\lambda|}+\mathrm{e}^{-\mathrm{i} t|\lambda|}\right) \mathrm{d} \lambda .
\end{aligned}
$$

The functional equation for the Eisenstein series and the fact that the scattering matrix $c_{\mu}(\mathrm{i} \lambda)$ is unitary for $\lambda \in \mathbb{R}$ imply that

$$
\sum_{k=1}^{K(\mu)} E\left(\phi_{k}^{\mu},-\mathrm{i} \lambda\right) \overline{E\left(\phi_{k}^{\mu},-\mathrm{i} \lambda\right)}=\sum_{k=1}^{K(\mu)} E\left(\phi_{k}^{\mu}, \mathrm{i} \lambda\right) \overline{E\left(\phi_{k}^{\mu}, \mathrm{i} \lambda\right)}
$$

from which (4.2) follows.

In the discussion below we will identify the operator $U^{\mu}(t)$ with its distributional kernel $U^{\mu}(\bullet) \in \mathcal{D}^{\prime}(\mathbb{R} \times \Gamma \backslash X \times \Gamma \backslash X)$.

We now follow a modification of Zelditch's argument [25] which in turn was inspired by Kuznecov's sum formulæ [17]. Thus, for two parabolic subgroups $P_{i}$ and $P_{j}$ we define

$$
\begin{aligned}
& U_{i j}^{\mu} \in \mathcal{D}^{\prime}\left(\mathbb{R} \times\left(\Gamma_{X_{P_{i}}} \backslash X_{P_{i}} \times A_{P_{i}}\right) \times\left(\Gamma_{X_{P_{j}}} \backslash X_{P_{j}} \times A_{P_{j}}\right)\right), \\
& U_{i j}^{\mu}(t)(x, y)=\int_{\Gamma_{N_{P_{i}}} \backslash N_{P_{i}} \Gamma_{N_{P_{j}}} \backslash N_{P_{j}}} U^{\mu}(t)\left(n x, n^{\prime} y\right) \mathrm{d} n \mathrm{~d} n^{\prime} .
\end{aligned}
$$

We note that $U_{i j}^{\mu}$ is the constant term of $U^{\mu}$, and hence the measures $\mathrm{d} n, \mathrm{~d} n^{\prime}$ have been normalized so that the total measures $\operatorname{vol}\left(\Gamma_{N_{P_{i}}} \backslash N_{P_{i}}\right), \operatorname{vol}\left(\Gamma_{N_{P_{j}}} \backslash N_{P_{j}}\right)$ are equal to 1.

For $0 \leqslant k_{1}, k_{2} \leqslant K(\mu)$, let $P_{i}, P_{j}$ be parabolic subgroups such that $\phi_{k_{1}}^{\mu} \in L^{2}\left(\Gamma_{X_{P_{i}}} \backslash X_{P_{i}}\right)$ and $\phi_{k_{2}}^{\mu} \in L^{2}\left(\Gamma_{X_{P_{j}}} \backslash X_{P_{j}}\right)$. For $a \in A_{P_{i}}, a^{\prime} \in A_{P_{j}}$ we now put

$$
u_{k_{1} k_{2}}^{\mu}\left(t, a, a^{\prime}\right)=\int_{\Gamma_{X_{P_{i}}} \backslash X_{P_{i}} \Gamma_{X_{P_{j}}} \backslash \int_{k_{P_{j}}}^{\mu} U_{i j}^{\mu}(t)\left(m a, m^{\prime} a^{\prime}\right) \overline{\phi_{k_{1}}^{\mu}(m)} \phi_{k_{2}}^{\mu}\left(m^{\prime}\right) \mathrm{d} m \mathrm{~d} m^{\prime},}^{\mu}
$$


noting that in the Riemann surface case discussed in [25], the space $\Gamma_{X_{P_{i}}} \backslash X_{P_{i}}$ reduces to a point, and only the terms with $\phi_{k_{1}}^{\mu}=\phi_{k_{2}}^{\mu}=1$ appeared.

Let us put

$$
\begin{aligned}
v_{k_{1} k_{2}}^{\mu}\left(t, a, a^{\prime}\right)= & \sum_{l=1}^{L(\mu)} \mathrm{e}^{\mathrm{i} t \sqrt{\lambda_{l}^{\mu}-\rho^{2}-\mu^{2}}} \int_{\Gamma_{X_{P_{i}}} \backslash X_{P_{i}}}\left(e_{l}^{\mu}\right)_{P_{i}}(m a) \overline{\phi_{k_{1}}^{\mu}(m)} \mathrm{d} m \\
& \times \int_{\Gamma_{X_{P_{j}} \backslash X_{P_{j}}} \overline{\left(e_{l}^{\mu}\right)_{P_{j}}\left(m^{\prime} a^{\prime}\right)} \phi_{k_{2}}^{\mu}\left(m^{\prime}\right) \mathrm{d} m^{\prime} .}
\end{aligned}
$$

Then $v_{k_{1}, k_{2}} \in \mathcal{C}^{\infty}\left(\mathbb{R} \times A_{P_{i}} \times A_{P_{j}}\right)$ and when we use (4.2), (3.3) and Proposition 3.7 in this definition we obtain

$$
\begin{aligned}
& u_{k_{1} k_{2}}^{\mu}\left(t, a, a^{\prime}\right)-v_{k_{1} k_{2}}^{\mu}\left(t, a, a^{\prime}\right) \\
& =\frac{1}{4 \pi} \sum_{k=1}^{K(\mu)} \int_{\mathbb{R}} \int_{\Gamma_{X_{P_{i}}} \backslash X_{P_{i}}} E_{P_{i}}\left(\phi_{k}^{\mu}, \mathrm{i} \lambda\right)(m a) \overline{\phi_{k_{1}}^{\mu}(m)} \mathrm{d} m \\
& \times \int_{\Gamma_{X_{P_{j}}} \backslash X_{P_{j}}} \overline{E_{P_{j}}\left(\phi_{k}^{\mu}, \mathrm{i} \lambda\right)\left(m^{\prime} a^{\prime}\right)} \phi_{k_{2}}^{\mu}\left(m^{\prime}\right) \mathrm{d} m^{\prime} \mathrm{e}^{\mathrm{i} t \lambda} \mathrm{d} \lambda \\
& =\frac{1}{4 \pi} \sum_{k=1}^{K(\mu)} \int_{\mathbb{R}} \mathrm{d} \lambda \int_{\Gamma_{X_{P_{i}}} \backslash X_{P_{i}}}\left(\delta_{i, j(k)} \mathrm{e}^{(\rho+\mathrm{i} \lambda) H} \phi_{k}^{\mu}(m)\right. \\
& \left.+\sum_{l=1}^{K(\mu)} \mathrm{e}^{(\rho-\mathrm{i} \lambda) H} c_{k l}(\mathrm{i} \lambda) \phi_{l}^{\mu}(m)\right) \overline{\phi_{k_{1}}^{\mu}(m)} \mathrm{d} m \\
& \times \int_{\Gamma_{X_{P_{j}}} \backslash X_{P_{j}}} \overline{\left(\delta_{j, j(k)} \mathrm{e}^{(\rho+\mathrm{i} \lambda) H^{\prime}} \phi_{k}^{\mu}\left(m^{\prime}\right)+\sum_{l=1}^{K(\mu)} \mathrm{e}^{(\rho-\mathrm{i} \lambda) H^{\prime}} c_{k l}(\mathrm{i} \lambda) \phi_{l}^{\mu}\left(m^{\prime}\right)\right)} \phi_{k_{2}}^{\mu}\left(m^{\prime}\right) \mathrm{d} m \\
& =\frac{1}{4 \pi} \sum_{k=1}^{K(\mu)} \int_{\mathbb{R}}\left(\delta_{k_{1} k} \mathrm{e}^{(\rho+\mathrm{i} \lambda) H}+\mathrm{e}^{(\rho-\mathrm{i} \lambda) H} c_{k k_{1}}(\mathrm{i} \lambda)\right) \overline{\left(\delta_{k k_{2}} \mathrm{e}^{(\rho+\mathrm{i} \lambda) H^{\prime}}+\mathrm{e}^{(\rho-\mathrm{i} \lambda) H^{\prime}} c_{k k_{2}}(\mathrm{i} \lambda)\right)} \mathrm{d} \lambda \\
& =\frac{1}{2 \pi} \int_{\mathbb{R}} \mathrm{e}^{\rho\left(H+H^{\prime}\right)}\left(\operatorname{Re} \mathrm{e}^{\mathrm{i} \lambda\left(H-H^{\prime}\right)} \delta_{k_{1} k_{2}}+\operatorname{Re}\left(\mathrm{e}^{-\mathrm{i} \lambda\left(H+H^{\prime}\right)} c_{k_{1} k_{2}}^{\mu}(\mathrm{i} \lambda)\right)\right) \mathrm{e}^{\mathrm{i} \lambda t} \mathrm{~d} \lambda,
\end{aligned}
$$

where $a=\mathrm{e}^{H}, a^{\prime}=\mathrm{e}^{H^{\prime}}$. In the second equation, we used the formula for the constant term $E_{P_{i}}\left(\phi_{k}^{\mu}, \mathrm{i} \lambda\right)$ in (3.3), and when $j(k) \neq i, \phi_{k}^{\mu}(m)$ is defined to be zero for $m \in \Gamma_{X_{P_{i}}} \backslash X_{P_{i}}$; in the third equation, we used the fact that $\phi_{k}^{\mu}$ are orthonormal eigenfunctions; and in the last equation, we used the fact that the scattering matrix $\left(c_{k_{1} k_{2}}^{\mu}(i \lambda)\right)$ is both unitary and symmetric for real $\lambda$ (see the comments after (3.5)).

We can summarize this discussion in 
PROPOSITION 4.1. - Let $s_{k_{1} k_{2}}^{\mu}(\lambda)=c_{k_{1} k_{2}}^{\mu}(-\mathrm{i} \lambda)$ be the entries of the scattering matrix defined in (3.3). Then for any $a=\mathrm{e}^{H} \in A_{P_{i}}$ and $a^{\prime}=\mathrm{e}^{H^{\prime}} \in A_{P_{j}}$ we have

$$
\begin{aligned}
\hat{s}_{k_{1} k_{2}}^{\mu} & \left(t-\left(H+H^{\prime}\right)\right)+\overline{\hat{s}_{k_{1} k_{2}}^{\mu}\left(-\left(H+H^{\prime}\right)-t\right)} \\
& -4 \pi \mathrm{e}^{-\rho\left(H+H^{\prime}\right)} u_{k_{1}, k_{2}}^{\mu}\left(t, a, a^{\prime}\right)+2 \pi\left(\delta_{0}\left(t+H-H^{\prime}\right)+\delta_{0}\left(t-H+H^{\prime}\right)\right) \delta_{k_{1} k_{2}} \\
& \in \mathcal{C}^{\infty}\left(\mathbb{R} \times A_{P_{i}} \times A_{P_{j}}\right),
\end{aligned}
$$

where $u_{k_{1} k_{2}}^{\mu}$ is defined by (4.4).

\section{Frequencies of the scattering matrix}

It is clear from Proposition 4.1 that the singularities of $\hat{s}_{k_{1} k_{2}}$ are directly related to the singularities of $u_{k_{1} k_{2}}^{\mu}$. That this relation is exact will follow from the following elementary

Lemma 5.1. - Suppose that $u \in \mathcal{C}^{\infty}\left(\left[r_{0}, \infty\right)_{r} ; \mathcal{D}^{\prime}\left(\mathbb{R}_{t}\right)\right)$ satisfies

$$
\operatorname{sing} \operatorname{supp} u(r, \bullet) \subset\left\{\ell_{j}+r\right\}_{j=0}^{\infty} \cup\left\{-\ell_{j}-r\right\}_{j=0}^{\infty}, \quad 0 \leqslant \ell_{j} \rightarrow \infty .
$$

If for $v, w \in \mathcal{D}^{\prime}(\mathbb{R})$ we have

$$
u(r, t)=v(t-r)+w(-t-r)
$$

then sing $\operatorname{supp} v \subset\left\{\ell_{j}\right\}_{j=0}^{\infty}$ and for $r_{1} \geqslant r_{0}$ such that $2 r_{0}-\ell_{j} \notin\left\{\ell_{i}\right\}_{i=0}^{\infty}$,

$$
v(x)-u\left(r_{1}, r_{1}+x\right) \in \mathcal{C}^{\infty}\left(\left(-\ell_{j}-\varepsilon_{j},-\ell_{j}+\varepsilon_{j}\right)_{x}\right), \quad \varepsilon_{j}>0 .
$$

Similarly, sing $\operatorname{supp} w \subset\left\{\ell_{j}\right\}_{j=0}^{\infty}$.

Proof. - Since $\left(\partial_{r}^{2}-\partial_{t}^{2}\right) u(r, t)=0$ for $r>0$ the lemma follows from the statement about propagation of singularities for the wave equation. More directly, we can make a change of variables, $x=t-r$ and $y=-(r+t)$, so that for $x+y \leqslant-2 r_{0}, \tilde{u}(x, y)=v(x)+w(y)$ and $\operatorname{sing} \operatorname{supp} \tilde{u} \subset\left\{x=\ell_{j}\right\} \cup\left\{y=\ell_{j}\right\}$. Then $v(x)=\tilde{u}(x, y)-w(y)$ and by choosing $y \neq \ell_{j}$ we see that sing $\operatorname{supp} v \subset\left\{\ell_{j}\right\}$. Also, for $y \notin\left\{\ell_{j}\right\}, v(x)-\tilde{u}(x, y) \in \mathcal{C}_{y}^{\infty}$.

We remark that the emergence of the one dimensional wave equation in this context is very natural and could be phrased in terms of the Lax-Phillips scattering theory [20].

Proposition 4.1 and Lemma 5.1 show that indeed, it suffices to study $u_{k_{1} k_{2}}^{\mu}\left(t, a, a^{\prime}\right)$, for a family of values of $a, a^{\prime}$. For that let us consider the decomposition of $\Gamma \backslash X$ given in Proposition 2.3. We will fix $r \gg 0$ and put

$$
B_{P, r}=\partial A_{P, r}=\left\{a \in A_{P} \mid \alpha(\log a)=r\right\},
$$

where $\alpha$ is the short root in $\Phi^{+}\left(P, A_{P}\right)$ if there are both short and long roots. We can arrange this so that $H=r$ for $a=\mathrm{e}^{H} \in B_{P, r}$. We then define as above (near Proposition 2.6) the sections at the height $r$ of the ends of $\Gamma \backslash X$ :

$$
Y_{P_{i}, r}=\Gamma_{P_{i}} \backslash N_{P_{i}} \times X_{P_{i}} \times B_{P_{i}, r} .
$$


Then for $a \in B_{P_{i}, r}$ and $a^{\prime} \in B_{P_{j}, r}$ we have

$$
u_{k_{1} k_{2}}^{\mu}\left(t, a, a^{\prime}\right)=\mathrm{e}^{2 \rho\left(H+H^{\prime}\right)} \frac{1}{v_{i} v_{j}} \int_{Y_{P_{i}, r}} \int_{Y_{P_{j}, r}} U(t)(x, y) \phi_{k_{1}}^{\mu}(m(x)) \phi_{k_{2}}^{\mu}\left(m^{\prime}(y)\right) \mathrm{d}_{Y_{P_{i}, r}} x \mathrm{~d}_{Y_{P_{j}, r}} y,
$$

where $\mathrm{d}_{Y_{P_{i}, r}} x$ and $\mathrm{d}_{Y_{P_{j}}, r} y$ are the induced Riemannian metrics on the closed embedded manifolds $Y_{P_{i}, r}$ and $Y_{P_{j}, r}$ in $\Gamma \backslash X$, and $v_{i}$ is the volume of $\Gamma_{N_{P_{i}}} \backslash N_{P_{i}}$ when the Riemannian metric of $N_{P_{i}}$ is induced from the identification of $N_{P_{i}}$ with the orbit $N_{P_{i}} x_{0}$ as a Riemannian submanifold in $X, v_{j}$ is similarly defined.

In the above equation, we used the following

LEMMA 5.2. - Let $f_{P_{i}}$ be given by (3.1). Then

$$
\begin{aligned}
& \left.\int_{\Gamma_{X_{P_{i}}} \backslash X_{P_{i}}} f_{P_{i}}(x)\right|_{\Gamma_{X_{P_{i}}} \backslash X_{P_{i}} \times B_{P_{i}, r}} \mathrm{~d} x \\
= & \int_{\Gamma_{X_{P_{i}}} \backslash X_{P_{i}}} \frac{1}{\operatorname{vol}\left(\Gamma_{N_{P_{i}}} \backslash N_{P_{i}} \times\{m\} \times B_{P_{i}, r}\right)} \int_{\Gamma_{N_{P_{i}}} \backslash N_{P_{i}} \times\{m\} \times B_{P_{i}, r}} f(n m) \mathrm{d} n \mathrm{~d} m \\
= & \int_{Y_{P_{i}, r}} \frac{1}{\operatorname{vol}\left(\Gamma_{N_{P_{i}}} \backslash N_{P_{i}} \times\{m\} \times B_{P_{i}, r}\right)} f(x) \mathrm{d}_{Y_{P_{i}}, r} x \\
= & \frac{1}{v_{i}} \mathrm{e}^{2 \rho r} \int_{Y_{P_{i}, r}} f(x) \mathrm{d}_{Y_{P_{i}, r}} x,
\end{aligned}
$$

where $m$ is any point of $X_{P_{i}}$, and the measure on $\Gamma_{N_{P_{i}}} \backslash N_{P_{i}} \times\{m\} \times B_{P_{i}, r}$ is the Riemannian density as a submanifold of $\Gamma_{N_{P_{i}}} \backslash N_{P_{i}} \times X_{P_{i}} \times A_{P_{i}}=\Gamma_{N_{P}} \backslash X$ and hence the total measure is equal to $\mathrm{e}^{-2 \rho r} v_{i}$.

The reason for the coefficient in front of the last integral is the fact that in the definition of the constant terms in (3.1), the total measure of $\Gamma_{N_{P_{i}}} \backslash N_{P_{i}}$ is normalized to be equal to 1 .

A statement about the singular support of $u_{k_{1} k_{2}}^{\mu^{i}}$ follows from the following general lemma which is a standard result about wave front sets.

LEMMA 5.3. - Let $M$ be a Riemannian manifold and $Y_{1}, Y_{2}$ two closed embedded submanifolds of $M$. Let $\Delta$ denote the (positive) Laplacian in $M$ and $\Phi^{t}$ the geodesic flow on $T^{*} M \backslash 0$. Let $\mathrm{d}_{Y_{P_{i}, r}}$ x be the induced Riemannian measure on $Y_{P_{i}, r}, i=1,2, A \in \Psi_{\mathrm{phg}}^{0}\left(M, \Omega_{M}^{1 / 2}\right)$, a classical pseudodifferential operator on $M$ and $\phi_{i} \in \mathcal{C}^{\infty}\left(Y_{i}\right), i=1,2$. If we define

$$
u(t)=\iint_{Y_{1}} \int_{Y_{2}}\left(A \mathrm{e}^{\mathrm{i} t \sqrt{\Delta}}\right)\left(x_{1}, x_{2}\right) \phi_{1}\left(x_{1}\right) \phi_{2}\left(x_{2}\right) \mathrm{d}_{Y_{1}} x_{1} \mathrm{~d}_{Y_{2}} x_{2}
$$

then

$$
\text { sing supp } u \subset \mathcal{T} \stackrel{\text { def }}{=}\left\{T_{j} \mid \Phi^{T_{j}}\left(N^{*} Y_{1}\right) \cap N^{*} Y_{2} \neq \emptyset\right\} .
$$

Proof. - We recall first the well known statement about propagation of singularities for the wave equation: if $U(t, x, y)=\exp (\mathrm{i} t \sqrt{\Delta})(x, y) \in \mathcal{D}^{\prime}(\mathbb{R} \times M \times M)$, then

$$
W F(U)=\left\{(t, x, y ; \tau, \xi, \eta) \in T^{*}(\mathbb{R} \times M \times M) \mid \tau=\sigma(\sqrt{\Delta})(x, \xi),(x, \xi)=\Phi^{t}(y, \eta)\right\} .
$$


Since $\tau \neq 0$ on $W F(A U)$ we conclude that $N^{*}\left(\mathbb{R} \times Y_{1} \times Y_{2}\right) \cap W F(A U)=\emptyset$ (we recall that the zero section of $T^{*} M$ is removed). Applying Corollary 8.2.7 of [13] we conclude that $\left.\widetilde{U} \stackrel{\text { def }}{=} A U\right|_{\mathbb{R} \times Y_{1} \times Y_{2}}$ is well defined and that

$$
\begin{gathered}
W F(\widetilde{U}) \subset\left\{\left(t, x_{1}, x_{2} ; \tau, \xi_{1}, \xi_{2}\right) \in T^{*}\left(\mathbb{R} \times Y_{1} \times Y_{2}\right) \mid \Phi^{t}\left(x_{2}, \tilde{\xi}_{2}\right)=\left(x_{1}, \tilde{\xi}_{1}\right),\right. \\
\left.\tau=\sigma(\sqrt{\Delta})\left(x_{1}, \tilde{\xi}_{1}\right), \pi_{1}\left(\tilde{\xi}_{1}\right)=\xi_{1}, \pi_{2}\left(\tilde{\xi}_{2}\right)=\xi_{2}\right\},
\end{gathered}
$$

where $\pi_{i}$ projects $T_{x_{i}}^{*} M$ to $T_{x_{i}}^{*} Y_{i}$ along $N_{x_{i}}^{*} Y_{i}$. The distribution $u(t)$ is just the pushforward of $\widetilde{U}$ along $Y_{1} \times Y_{2}$ and consequently, by Theorem 8.2.12 of [13], obtain

$$
W F(u) \subset\left\{(t, \tau) \mid \exists\left(x_{1}, \xi_{1}\right) \in N^{*} Y_{1},\left(x_{2}, \xi_{2}\right) \in N^{*} Y_{2}, \text { such that } \Phi^{t}\left(x_{2}, \tilde{\xi}_{2}\right)=\left(x_{1}, \tilde{\xi}_{1}\right)\right\} .
$$

The lemma is an immediate consequence.

Refinements of this bring us into the framework of "Kuznecov formulæ" for manifolds studied in [25]. It is clear that the precise description of singularities of $u(t)$ under further geometric assumptions follows from the calculus of Lagrangian distributions of Hörmander - see [12], [6] and Section 25.2 of [14]. In fact, as carried out in [25] (see Proposition 1.10 there), the calculus of Fourier Integral Operators gives the following

LEMma 5.4. - Let $u(t)$ be the distribution defined in Lemma 5.3. If we assume in addition that

$$
\Phi^{t}\left(N^{*} Y_{1}\right) \text { intersects } N^{*} Y_{2} \text { cleanly for all times } t,
$$

and for $T_{j} \in \mathcal{T}$ define,

$$
W_{j}=\left\{\Phi^{t}(x, \xi) \in T^{*} M|0 \leqslant t \leqslant| T_{j} \mid, \quad(x, \xi) \in N^{*} Y_{1} \cap \Phi^{-T_{j}}\left(N^{*} Y_{2}\right)\right\},
$$

assuming (for simplicity only) that $W_{j}$ is connected then,

$$
u(t)=(2 \pi)^{-\frac{e_{j}}{2}} \mathrm{e}^{\frac{\mathrm{i} \pi}{4} m_{j}} \sum_{l=0}^{\infty} \sigma_{j l}\left(t-T_{j}+\mathrm{i} 0\right)^{-\frac{1}{2} e_{j}-\frac{1}{2}+l} \bmod \mathcal{C}^{\infty}, \quad t \in\left(T_{j}-\varepsilon_{j}, T_{j}+\varepsilon_{j}\right),
$$

where $e_{j}=\operatorname{dim} W_{j}-2, m_{j} \in \mathbb{Z}$, the Maslov index of $W_{j}$ and

$$
\sigma_{j 0}=\int_{N^{*} Y_{1} \cap \Phi^{-T_{j}}\left(N^{*} Y_{2}\right) \cap S^{*} M} \pi^{*} \phi_{1}(m)\left(\Phi^{-T_{j}}\right)^{*} \pi^{*} \phi_{2}(m) \sigma(A)(m) \mathrm{d} \mu_{j}(m), \quad \pi: N^{*} Y_{1} \rightarrow Y_{1} .
$$

Here $\mathrm{d} \mu_{j}$ is a natural density defined in (2.6.1) of [25] (see also (5.7) below).

To compute the principal symbol of $\hat{s}_{k_{1} k_{2}}^{\mu}$ we need to review the construction of the density $\mathrm{d} \mu_{j}$ on $N^{*} Y_{1} \cap \Phi^{-T_{j}}\left(N^{*} Y_{2}\right) \cap S^{*} M$ and for that we follow [6] (or Section 21.6 and Section 25.2 of [14]) as in Section 2(a) of [25]. Since the construction is quite involved we review the construction. The half-density computations provide an invariant description of the symbols - in local coordinates and in direct representation of Fourier Integral Operators, they correspond to computing the Hessian factors in applications of the stationary phase method.

An $a$-density on a $k$-dimensional vector space $W$, is a map $f: \bigwedge^{k} W \rightarrow \mathbb{R}_{+}$, satisfying $f(t v)=|t|^{a} f(v)$. The space of $a$-densities on $W$ is denoted by $\Omega^{a}(W)$. For a choice of a basis and with the corresponding coordinates $w=\left(w_{1}, \ldots, w_{k}\right)$, we write an $a$-density as $\alpha|\mathrm{d} w|^{a}$. 
Let $V_{j} \subset V, j=1,2$, be two Lagrangian subspaces of a symplectic vector space $V$. We then have a short exact sequence

$$
\begin{gathered}
0 \longrightarrow V_{1} \cap V_{2} \stackrel{\imath}{\longrightarrow} V_{1} \oplus V_{2} \stackrel{\tau}{\longrightarrow} V \longrightarrow V / \operatorname{Im} \tau \longrightarrow 0, \\
\imath(v)=(v, v), \quad \tau\left(v_{1}, v_{2}\right)=v_{1}-v_{2} .
\end{gathered}
$$

Then,

$$
\Omega^{1 / 2}\left(V_{1} \cap V_{2}\right) \simeq \Omega^{1 / 2}\left(V_{1} \oplus V_{2}\right) \otimes \Omega^{-1 / 2}(V) \otimes \Omega^{1 / 2}(V / \operatorname{Im} \tau),
$$

where the identification is determined by $\imath$ and $\tau$. It is easy to visualize it in the coordinates in which the maps take the form $\mathbb{R}^{k} \rightarrow \mathbb{R}^{k} \times \mathbb{R}^{2 n-k} \rightarrow \mathbb{R}^{2 n-k} \times \mathbb{R}^{k} \rightarrow \mathbb{R}^{k}$, successively as $v \mapsto(v, 0),(v, w) \mapsto(w, 0),\left(v^{\prime}, w\right) \mapsto v^{\prime}$ :

$$
|\mathrm{d} v|^{1 / 2}=|\mathrm{d} v \mathrm{~d} w|^{1 / 2}\left|\mathrm{~d} w \mathrm{~d} v^{\prime}\right|^{-1 / 2}\left|\mathrm{~d} v^{\prime}\right|^{1 / 2} .
$$

The symplectic form identifies $\left(V_{1} \cap V_{2}\right)^{*}$ with $V / \operatorname{Im} \tau$ since

$$
(\operatorname{Im} \tau)^{\perp}=\left(V_{1}+V_{2}\right)^{\perp}=V_{1}^{\perp} \cap V_{2}^{\perp}=V_{1} \cap V_{2},
$$

where we used the fact that $V_{j}$ 's are Lagrangian: $V_{j}^{\perp}=V_{j}$. Hence, with this identification, $\Omega^{1 / 2}(V / \operatorname{Im} \tau) \simeq \Omega^{1 / 2}\left(\left(V_{1} \cap V_{2}\right)^{*}\right) \simeq \Omega^{-1 / 2}\left(V_{1} \cap V_{2}\right)$, where the last isomorphism is canonical. Finally,

$$
\Omega^{1}\left(V_{1} \cap V_{2}\right) \simeq \Omega^{1 / 2}\left(V_{1}\right) \otimes \Omega^{1 / 2}\left(V_{2}\right) \otimes \Omega^{-1 / 2}(V),
$$

where the isomorphism depends on $i, \tau$ and the symplectic structure.

Using this identification, we can now define the density appearing in Lemma 5.4:

$$
\begin{gathered}
\tilde{\mu}_{j}=\frac{\sigma_{1} \otimes\left(\Phi^{T_{j}}\right)^{*} \sigma_{2}}{\left|\omega_{T^{*} M}^{n}\right|^{1 / 2}}, \quad \mu_{j}=\widetilde{\mu}_{j} \otimes|\mathrm{d} q|^{-1}, \\
V_{1}=T_{p}\left(N^{*} Y_{1}\right), \quad V_{2}=T_{p}\left(\Phi^{-T_{j}}\left(N^{*} Y_{2}\right)\right), \quad V=T_{p}\left(T^{*} M\right), \\
\sigma_{j} \in C^{\infty}\left(N^{*} Y_{j} ; \Omega^{1 / 2}\left(T\left(N^{*} Y_{j}\right)\right)\right), \text { is induced by the Riemannian metric on } M, \\
q \text { is the metric on the fibers of } T^{*} M, \\
\omega \text { is the symplectic form on } T^{*} M, \quad n=\operatorname{dim} M .
\end{gathered}
$$

We will now use Proposition 2.11 to describe this half density in our setting:

LEMma 5.5. - When $M=\Gamma \backslash X, Y_{1}=Y_{P_{i}, r}, Y_{2}=Y_{P_{j}, r}, T \in \mathcal{T}=\left\{T_{j} \mid \Phi^{T_{j}}\left(N^{*} Y_{1}\right) \cap\right.$ $\left.N^{*} Y_{2} \neq \emptyset\right\}$ and $T \neq 0$, then, under the identification between $N^{*} Y_{P_{i}, r} \cap \Phi^{-1}\left(N^{*} Y_{P_{j}, r}\right) \cap$ $S^{*}(\Gamma \backslash X)$ with $X_{T, i j}$ given by (2.4), the density $\mu_{T}$ in (5.7) is given by

$$
\mu_{T}=\mathrm{e}^{-|T| \rho}\left(\pi_{T, i}\right)^{*} \mathrm{~d} g_{\Gamma_{X_{P_{i}}} \backslash X_{P_{i}}}
$$

where $\mathrm{d} g_{\Gamma_{X_{P_{i}}} \backslash X_{P_{i}}}$ is the Riemannian density, and

$$
\rho=\rho_{P_{j}}=\rho_{P_{i}}
$$

is the half sum of roots in $\Phi^{+}\left(P_{j}, A_{P_{j}}\right)$ and is identified with a number in $\mathbb{R} \simeq \mathfrak{a}_{P_{j}}$. 
Proof. - On the universal covering space $X$, scattering geodesics always scatter between two opposite parabolic subgroups with respect to some common split component (see the proof of Proposition 2.9), we can assume that $P_{i}$ and $P_{j}$ are opposite parabolic subgroups. We observe that if $T=0$, the density $\mu$ is equal to the Riemannian density on $N^{*} Y_{P_{i}, r} \cap N^{*} Y_{P_{j}, r} \cap$ $S^{*}(\Gamma \backslash X) \equiv X_{T, m l}$ (see Diagram (2.4) for the identification of the spaces). The reason is that all the densities are canonically induced from the Riemannian metric of $\Gamma \backslash X$. Assume next $T>0$. Under the geodesic flow $\Phi^{T}, \Phi^{T}\left(N^{-} \widetilde{Y}_{P_{j}, r}\right)=N^{-} \widetilde{Y}_{P_{j}, r-T}$. Hence, all the horospheres $\widetilde{Y}_{P_{j}, r}$ of $P_{j}$ can be identified under the geodesic flow. Let $d y$ be the Riemannian density of the horosphere $\widetilde{Y}_{P_{j}, 0}$ as a submanifold of $X$. Then the Riemannian density of the horosphere $\widetilde{Y}_{P_{j}, r}$ is equal to $\mathrm{e}^{-2 r \rho} \mathrm{d} y$. This implies that the pushed forward density $\left(\Phi^{T}\right)^{*} \sigma_{2}$ on $Y_{P_{j}, r-T}$ is equal to the half Riemannian density of $Y_{P_{j}, r-T}$ times $\mathrm{e}^{-T \rho}$. Combined with the above observation, this proves Lemma 5.5.

We now have all the components needed to prove Theorem 2.

Proof of Theorem 2. - As stated in the beginning of this section, we need to understand the singularities of $u^{\mu}\left(t, a, a^{\prime}\right)$ defined in (4.4). First, we notice that the modified wave kernel,

$$
\cos t \sqrt{\Delta-\rho^{2}-\mu}=\frac{1}{2}\left(\mathrm{e}^{\mathrm{i} t \sqrt{\Delta-\rho^{2}-\mu}}+\mathrm{e}^{-\mathrm{i} t \sqrt{\Delta-\rho^{2}-\mu}}\right)
$$

is a sum of two terms of the form $A \mathrm{e}^{ \pm \mathrm{i} \sqrt{\Delta}}$, discussed in Lemma 5.3, with the principal symbol of $A$ equal to $1 / 2$. Using Lemma 5.2, we can apply Lemma 5.4 (with Maslov indices $m_{j}=0$ since we are on a negatively curved manifold), and Lemma 5.5 to $u^{\mu}\left(t, a, a^{\prime}\right)$. Let us fix $a$ and $a^{\prime}$ so that $H=H^{\prime}=r$. The propagation lemma (Lemma 5.3) shows that the singularities of $u^{\mu}\left(t, a, a^{\prime}\right)$ are contained in the set $\mathcal{T}=\left\{T_{k}\right\}$, and a precise statement about the structure of the singularity comes from (5.5): the trace involving our modified propagator corresponds to $(u(t)+\overline{u(t)}) / 2$.

To understand the singularities of the scattering matrix $\left(\hat{s}_{k_{1} k_{2}}\right)$, we consider two cases. When $k_{1} \neq k_{2}$, Proposition 4.1 and Lemma 5.1 show that the singularities of $\hat{s}_{k_{1} k_{2}}(t-2 r)$ are determined by the singularities $u_{k_{1} k_{2}}^{\mu}\left(t, a, a^{\prime}\right)$. By Proposition 2.11, when the parabolic subgroups $P_{i}, P_{j}$ for $\phi_{k_{1}}^{\mu}, \phi_{k_{2}}^{\mu}$ are different, the set $\mathcal{T}$ in Lemma 5.5 does not contain zero, and $\left\{T_{k}-2 r \mid T_{k} \in \mathcal{T}, T_{k}>0\right\}$ is exactly the set $\mathcal{T}_{i j}$ of sojourn times of scattering geodesics between $P_{i}$ and $P_{j}$. Then it follows from Lemma 5.3 that the singularities of $u_{k_{1} k_{2}}^{\mu}\left(t, a, a^{\prime}\right)$ are included in the set $\left\{T+2 r \mid T \in \mathcal{T}_{i j}\right\} \cup\left\{-T-2 r \mid T \in \mathcal{T}_{i j}\right\}$. Therefore, by Lemma 5.1, the singularities of $\hat{s}_{k_{1} k_{2}}(t)$ are exactly located at the set $\mathcal{T}_{i j}$ of sojourn times. To get the structure of the singularities, we notice that the factors $\mathrm{e}^{-\rho\left(H+H^{\prime}\right)}$ in Proposition 4.1, $\mathrm{e}^{2 \rho\left(H+H^{\prime}\right)}$ in (5.2) and $\mathrm{e}^{-T_{k} \rho}$ in Lemma 5.5 combine into $\mathrm{e}^{-\left(T_{k}-2 r\right) \rho}$, where $T_{k}-2 r$ is a sojourn time as mentioned earlier. On the other hand, when the parabolic subgroups $P_{i}, P_{j}$ are the same, the set $\mathcal{T}$ in Lemma 5.5 contains zero. By Lemma 5.4 and a variant of Lemma 5.5, this point $T_{k}=0$ does not contribute to the singularities of $u_{k_{1} k_{2}}^{\mu}\left(t, a, a^{\prime}\right)$ since $\phi_{k_{1}}^{\mu}, \phi_{k_{2}}^{\mu}$ are orthogonal to each other - see the description of the propagator $U^{\mu}(t)$ in terms of Eisenstein series (4.2), and their construction in Lemma 3.5 (and also (3.3)).

For the case $k_{1}=k_{2}$, there is an additional singularity at $t=0$ given by $\delta_{0}(t)=\delta_{0}(t) \delta_{k_{1} k_{2}}$ in Proposition 4.1: the set $\mathcal{T}$ in Lemma 5.5 does contain zero. The singularity of $u_{k_{1} k_{2}}^{\mu}\left(t, a, a^{\prime}\right)$ at $t=0$ is nontrivial and cancels out the singularity of $\delta_{0}(t)$. The easiest way to see that is to suppose that the singularities do not cancel out. Then, Proposition 4.1 shows that $\hat{s}_{k_{1} k_{2}}^{\mu}(t)$ is singular at either $2 r$ or $-2 r$. Since $r$ is arbitrary, this is impossible. In fact, (4.5) applied with a fixed $r\left(H=H^{\prime}=r\right)$ shows that the singularities of the left hand side form a discrete set. Same argument as before shows that the singularities of $\hat{s}_{k_{1} k_{2}}(t)$ are located at 
$\left\{T_{k}-2 r \mid T \in \mathcal{T}, T_{k}>0\right\}$, which is the set of sojourn times $\mathcal{T}_{i j}$. The structure of the singularities can be determined as in the case $k_{1} \neq k_{2}$.

\section{Acknowledgments}

The authors would like to thank the National Science Foundation of the U.S. and the National Science and Engineering Research Council of Canada for partial support. The first author is partially supported by an Alfred P. Sloan research fellowship. Thanks go also to Steve Zelditch for helpful comments on the first version of this paper, and to the referee for suggestions which lead to several improvements and corrections.

\section{REFERENCES}

[1] BoREL A., Introduction aux groupes arithmétiques, Hermann, Paris, 1969.

[2] Borel A., Linear algebraic groups, in: Proc. of Symp. in Pure Math., Vol. 9, pp. 3-19.

[3] Borel A. Reduction theory for arithmetic subgroups, in: Proc. of Symp. in Pure Math., Vol. 9, pp. 2025.

[4] Borel A., Serre J.-P., Corners and arithmetic groups, Comment. Math. Helv. 48 (1973) 436-491.

[5] Borel A., Tits J., Groupes réductifs, I.H.E.S. 27 (1965) 55-150.

[6] Duistermaat J., Guillemin V., The spectrum of positive elliptic operators and periodic bicharacteristics, Inv. Math. 29 (1975) 39-79.

[7] Freitag E., Hilbert Modular Forms, Springer-Verlag, 1990.

[8] Gelfand I.M., Piatetski-Shapiro I.I., Unitary representations in homogeneous spaces with discrete stationary groups, Soviet Math. Dokl. 3 (1962) 1528-1531.

[9] GUILLEMIN V., Sojourn times and asymptotic properties of the scattering matrix, Publ. RIMS, Kyoto Univ. 12 (1977) 69-88 (Suppl.).

[10] GUILLEMIN V., Notes on scattering theory, MIT, 1976, unpublished notes.

[11] Harish-Chandra, Automorphic Forms on Semisimple Lie Groups, Lect. Notes in Math., Vol. 62, 1968.

[12] Hörmander L., Fourier integral operators I, Acta Math. 127 (1971) 79-183.

[13] Hörmander L., The Analysis of Linear Partial Differential Operators, Vol. I, Springer-Verlag, Berlin, 1983.

[14] Hörmander L., The Analysis of Linear Partial Differential Operators, Vol. IV, Springer-Verlag, Berlin, 1985.

[15] JI L., The Weyl upper bound on the discrete spectrum of locally symmetric spaces, J.D.G. 51 (1999) 97-147.

[16] Ji L., MCPHERSON R., Geometry of compactifications of locally symmetric spaces, preprint, 1997.

[17] KuZneCov V.N., Petersson's conjecture for cusp forms of weight 0 and Linnik's conjecture: sums of Kloosterman sums, Math. USSR Sbornik 39 (1981).

[18] Langlands R., On the Functional Equations Satisfied by Eisenstein Series, Lect. Notes in Math., Vol. 544, 1976.

[19] LANGLANDS R., Eisenstein series, the trace formula, and the modern theory of automorphic forms, in: Number Theory, Trace Formula and Discrete Groups, Academic Press, Boston, MA, 1989, pp. 125155.

[20] LaX P., PhILlips R., Scattering Theory for Automorphic Functions, Princeton University Press, 1978.

[21] MÜLlER W., The trace class conjecture in the theory of automorphic forms, Ann. of Math. 130 (1989) 473-529.

[22] Petkov V., Stoyanov L., Geometry of Reflecting Rays and Inverse Spectral Problems, Wiley, 1992.

[23] SAPER L., Tilings and finite energy retractions of locally symmetric spaces, Comment. Math. Helv. 72 (1997) 167-202. 
[24] Selberg A., On discontinuous groups in higher dimensional symmetric spaces, in: Contributions to Function Theory, Tata Inst. of Fund. Res., Bombay, 1960.

[25] Zelditch S., Kuznecov sum formulæ and Szegö limit formulæ on manifolds, Comm. P.D.E. 17 (1992) 221-260.

(Manuscript received December 15, 1999; accepted, after revision, October 26, 2000.)

\author{
Lizhen JI \\ Department of Mathematics, \\ University of Michigan, \\ Ann Arbor, MI 48109, USA \\ E-mail: 1ji@math.lsa.umich.edu \\ Maciej ZWORSKI \\ Mathematics Department, \\ University of California, \\ Berkeley, CA 94720, USA \\ E-mail: zworski@math.berkeley.edu
}

